\title{
DYNAMIC LATERAL StabILITY OF Elastomeric SEISMIC
}

Abstract Predicting the response of elastomeric seismic isolation bearings when subjected to severe ground motions is challenging due to the highly nonlinear behavior associated with the bearings under a combination of large displacements and axial loads. In particular, the horizontal stiffness of the bearings is a function of both horizontal displacement as well as axial load that varies due to overturning moments. Previous analytical models or formulations to model these bearings were mainly developed to estimate critical loads at the stability limit. Only few of these models are capable of estimating the correct nonlinear behavior of bearings observed at horizontal displacements in excess of the bearing width. In this study, a nonlinear analytical model is presented that is capable of modeling the dynamic response of bearings more accurately at all displacement ranges, especially beyond the stability limit and is verified with experimental data from an earlier experimental study. It was observed in the dynamic experiments that the bearings have a far larger capability to sustain horizontal loads at displacements exceeding their stability limit than predicted by earlier models and more importantly the bearings re-centered after these large displacement excursions. This behavior is captured using the analytical model developed in this 17 study.

\section{INTRODUCTION}

Base isolation has become a widely accepted technique in structural engineering over the past three decades for protecting structures from severe ground motion. Lead rubber bearings and spherical sliding

\footnotetext{
${ }^{1}$ PhD Candidate, Rice University, Houston, TX - 77005, USA, svv1@ rice.edu

${ }^{2}$ Professor, Rice University, Houston, TX-77005, USA, satish.nagarajaiah@rice.edu

* corresponding author

${ }^{3}$ PhD Candidate, University at Buffalo (SUNY), Buffalo, NY - 14260, USA, am249@ buffalo.edu

${ }^{4}$ Associate Professor, University of California, San Diego, CA - 92093, USA, gmosqueda@ucsd.edu
} 
21 bearings constitute the most widely used seismic protection technology. Due to their inherent flexibility in

22 the horizontal direction, the bearings have the capacity to undergo large displacements when subjected to

23 strong ground motion. Under a combination of large displacements and varying axial loads, the behavior

24 of elastomeric bearing becomes highly nonlinear. Under such circumstances the horizontal stiffness of the

25 bearings becomes a nonlinear function of axial load and lateral displacement.

26 The theoretical approaches adopted by researchers to address stability of elastomeric bearings (Derham \&

27 Thomas, 1981; Gent, 1964) made use of Haringx's theory (Haringx, 1948; Haringx, 1949a; Haringx,

28 1949b) of flexible columns. Both the studies predicted decrease in horizontal stiffness of the bearings

29 with increasing axial load. Buckle and Kelly (1986) conducted experimental studies on a scaled bridge

30 model equipped with slender elastomeric bearings. Koh and Kelly (1988) developed a two-spring

31 mechanical model that takes into account the influence of axial load on horizontal stiffness of bearings,

32 and also a viscoelastic stability model (Koh and Kelly, 1989) for elastomeric bearings. The use of

33 Haringx's type formulation for modeling the stiffness of elastomeric bearings is found to be closer to

34 experimental results (Bažant, 2003; Bažant \& Cedolin, 1991).

35 Nagarajaiah and Ferrell (1999) proposed an enhanced nonlinear model based on the linear two-spring 36 model developed by Koh and Kelly (1988). In their study the authors demonstrate the ability of the

37 analytical model to capture the force-displacement behavior of elastomeric bearings when subjected to

38 large axial loads and horizontal displacements. Further they also show that the critical load of the bearings

39 reduced with increasing horizontal displacement and the horizontal stiffness decreases with increasing

40 horizontal displacement and axial load. Nagarajaiah and Ferrell (1999) validated and verified their

41 analytical model using results from the experimental study performed by Buckle and co-wokers. These

42 experimental results were later documented in the paper by Buckle et al. (2002). Iizuka (2000) proposed a

43 macroscopic model for predicting the response of laminated rubber bearings at large deformations. The

44 model proposed by lizuka (2000) was also a modified version of the two-spring model proposed by Koh

45 and Kelly (1989), where the linear springs were replaced with non-linear springs. The author Iizuka 
46 (2000) determines the nonlinear parameters of the rotational and shear spring empirically from results of

47 basic load testing on laminated rubber bearings. Kikuchi et al. (2010) developed a new analytical model

48 comprising of multiple shear springs at the mid-height and a series of axial springs at the top and bottom

49 boundaries of the model. Their work predominantly focuses on square seismic isolation bearings and they

50 highlight the importance loading direction has on the ultimate behavior of the bearings. Weisman and

51 Warn (2012) present experimental testing and detailed nonlinear finite element analysis for investigating

52 the critical load capacities of elastomeric and lead-rubber bearings at large lateral displacements. They

53 performed a parametric study to investigate the dependency of critical load on bearing geometry (Warn

54 and Weisman 2011). Cardone and Perrone (2012) also reported critical loads from experiments performed

55 on slender elastomeric bearings.

56 A recent study by Sanchez et al. (2012) focuses on an experimental testing program to examine the

57 behavior of elastomeric bearings at and beyond their stability limit. Based on quasi-static tests performed

58 on three different types of reduced scale elastomeric bearings, the authors conclude that the reduced area

59 formula (Sanchez et al. 2012) based on effective shear modulus at $25 \%$ shear strain is more accurate in

60 predicting the critical loads of bearings. The authors also note the ability of the bearings to recover from

61 motions exceeding their stability limits during dynamic tests and identify the critical load and shear strain

62 limits below which instability in the bearings is unlikely to occur. Han et al. (2013) studied in a detailed

63 manner the controlling mechanism that governs the critical loads in elastomeric bearings. They compare

64 the capability of two different analytical models: Nagarajaiah and Ferrell (1999) and Iizuka (2000)

65 models, for predicting the critical loads and displacements of elastomeric isolation bearings. They

66 perform a global sensitivity analysis on the model parameters and identify that the prediction is most

67 sensitive to the nonlinear behavior of the rotational spring for lateral displacements greater than 0.6 times

68 bearing diameter/width. Han et al. (2013) propose a modified analytical model based on the sensitivity

69 analysis using fewer empirical parameters that has similar predictive capabilities as that of Iizuka (2000)

70 model. 
71 While recent analytical studies have provided considerable insight into the nonlinear behavior of bearings,

72 instances of rollover or instability were observed during pervious experimental studies. Buckle and Kelly

73 (1986) experimentally studied the dynamic performance of slender elastomeric bearings in an isolated

74 bridge deck model. The bearings were dowelled and experienced partial and complete rollover during

75 dynamic testing. Griffith et al. (1987) conducted experiments on a quarter scale nine-story isolated test

76 model to study the effectiveness of an uplift restraint device. In certain test cases where restraints were

77 not installed, column uplift was observed and the force-displacement loops of supporting bearing appear

78 to become unstable due to a sudden drop in stiffness.

79 Particularly for performance-based design, it is important to extend the theoretical understanding on the

80 stability of elastomeric bearings based on static/quasi-static tests to dynamic behavior and enhance our

81 ability to predict their response when subjected to extreme earthquake loading. In this study an enhanced

82 analytical model is developed based on the nonlinear model developed by Nagarajaiah and Ferrell (1999)

83 and its effectiveness in predicting the dynamic response of elastomeric bearings is evaluated and verified

84 using experimental data from the study by Sanchez et al (2012). The key contribution of this study is to

85 develop a detailed analytical model that is capable of modeling the nonlinear response of elastomeric

86 bearings under extreme loads, including the capability to more accurately capture its dependence on

87 horizontal displacement and axial loads at displacements exceeding the stability limit. The findings of this

88 study are in clear agreement with recently reported observations by Han et al. (2013) that the rotational

89 spring stiffness of the analytical model is the governing factor at large displacements. A new analytical

90 model to capture the exact nature of this nonlinearity is proposed in this study.

\section{NONLINEAR ANALYTICAL BEARING MODEL}

92 Figure 1 shows the nonlinear analytical model developed to model the behavior of elastomeric bearings in

93 the two-dimensional plane. It is based on the Koh and Kelly (1986) linear model, and was first enhanced

94 and developed into a nonlinear form by Nagarajaiah and Ferrell (1999). In this study the shear and the

95 rotational springs of the analytical model are considered to be nonlinear elastic. The nonlinearities are 

model to predict behavior of the bearings beyond the stability limit at large displacements and axial loads. model considered has two degrees of freedom (DOF), the shear displacement, $s$, governed by the nonlinear shear spring, $K_{s}$, and rotation, $\theta$, governed by nonlinear rotational springs of stiffness, $K_{\theta} / 2$. The model is subjected to axial load, $P$, and horizontal load, $F$, at the top of the bearing. The top plate is free to move in both horizontal and vertical directions but restrained from rotating. When the bearing displaces in the horizontal direction by an amount $u$, it is a result of a shear displacement, $s$, and rotation, $\theta$, of the analytical model. The horizontal displacement, $u$, is given by the relation

$$
u=l \sin \theta+s \cos \theta
$$

108 Where, $l$ is the combined height of rubber layers and steel shims. The nonlinear horizontal stiffness of the model, $K_{h}$, is a function of the axial load, $P$, and the horizontal displacement, $u$. In the nonlinear analytical model, both the shear stiffness, $K_{s}$, and the rotational stiffness, $K_{\theta}$, vary as a function of the shear deformation, $s$.

\section{Equilibrium equations}

113 The equilibrium equations of the analytical model shown in Figure 1 are given by

$$
K_{S} S=F \cos \theta+P \sin \theta+\frac{K_{\theta o} \delta C_{\theta} \theta^{2}}{2}
$$

$$
K_{\theta} \theta=P(l \sin \theta+s \cos \theta)+F(l \cos \theta-s \sin \theta)
$$


Where $K_{s}$ refers to the nonlinear shear stiffness of the model, $K_{\theta}$ is the nonlinear rotational stiffness of the model, $\delta$ is a constant of value 1 and dimensions $(1 / \mathrm{mm})$ and $K_{\theta o}$ is the nonlinear rotational stiffness of the model at zero shear displacement..

121 In the analytical model the estimated variation of shear modulus, $G$, with horizontal displacement is 122 mainly captured using the variation of the nonlinear shear stiffness, $K_{s}$, with respect to shear deformation, $123 s$.

$$
K_{s}=K_{s o}\left(1-C_{s} \tanh \left(\alpha \frac{s}{l_{r}}\right)\right)
$$

125

where $K_{s o}$ refers to the shear stiffness at zero shear strain, $C_{s}$ is a dimensionless constant, and $\alpha$ is a dimensionless constant with a value of $l_{r}$. In order to account for correct axial-load horizontal displacement behavior the nonlinear rotational stiffness of the model, $K_{\theta}$, is considered a function of $s / l_{r}$ by Nagarajaiah and Ferrell (1999).

$$
K_{\theta}=K_{\theta o}\left(1-C_{\theta} \frac{s}{l_{r}}\right)
$$

where $K_{\theta o}$ refers to the nonlinear rotational stiffness at zero shear strain and $C_{\theta}$ is a dimensionless constant.

The horizontal stiffness of the bearings $K_{h}$ is a function of horizontal displacement, $u$, and axial load, $P$. The equilibrium paths for a given set of input parameters $\left(C_{s}, C_{\theta}, K_{s o}\right.$, and $\left.K_{\theta o}\right)$ are solved using RungeKutta method to obtain values of $s$ and $\theta$ corresponding to the applied horizontal load, $F$, and vertical load, $P$. The ability of the proposed analytical model to predict the behavior of elastomeric bearings when subjected to seismic loads is evaluated in the sections that follow.

\section{EXPERIMENTAL RESULTS}

Experiments by Sanchez et al. (2012) examined the stability limit of four different types of elastomeric bearings using the University of Buffalo NEES equipment site. Three of the types of bearings are low damping natural rubber bearings and the fourth include a lead plug. The bearings were subjected to both 
141 quasi-static and dynamic tests, the main emphasis of the experimental verifications done in this paper 142 focus on dynamic tests. More details on the quasi-static experimental program can be found in Sanchez 143 (2010) and Sanchez et al. (2012) while Masroor et al. (2012) provide detailed results on the dynamic 144 stability tests. Among the bearing test results considered, six bearings belong to the same category with 145 two subjected to quasi-static tests and the remaining four subjected to dynamic tests. The properties of the 146 six bearings obtained from initial characterization tests are listed in Table 3 . The properties listed include 147 the effective shear modules, $G_{e f f}$, and the effective damping ratio, $\beta$, computed from $0.1 \mathrm{~Hz}$ cyclic test 148 data at $100 \%$ shear strain for two different axial loads. This data provides some insight into the 149 dependence of the bearing behavior on axial load and also the variation in bearing properties for the six 150 nominally identical bearings.

\section{Quasi-static stability tests}

152 Quasi-static stability tests were performed on the bearings using the Single Bearing Test Machine 153 (SBTM) designed by Sanchez et al. (2012). The test setup has the ability to simultaneously apply

154 horizontal deformations and axial loads. Two different testing methods were used to evaluate the stability 155 of bearings. Method 1 applied a predetermined initial displacement to the bearing that remains constant 156 while the axial load is increased monotonically from zero to a point where the horizontal force resistance 157 of the bearing becomes zero. This approach was first proposed by Buckle et al. (2002) and Nagarajaiah 158 and Ferrell (1999). In Method 2, a predetermined initial axial load is applied to the bearing and the 159 horizontal displacement of the bearing is increasing monotonically from zero to a point where the 160 horizontal force resistance of the bearing becomes zero. Only the results from Method 2 are considered 161 here. The results obtained using Method 2 for two different bearings (labeled 15180 and 15196) are 162 shown in Figure 2. The nonlinear nature of the force displacement curves is clearly evident from the 163 figure and in addition it is also apparent there exists considerable variation in experimental results from 164 two nominally identical bearings. 


\section{Dynamic Tests}

166

167

168

169

170

171

172

173

174

175

176

177

178

179

180

181

182

183

184

185

186

187

The dynamic stability tests, (Sanchez et al. 2012) subjected a rigid mass supported on four bearings shown in Figure 3 to unidirectional extreme ground motions, driving the system beyond the stability limit. Though instability in bearings was earlier encountered unexpectedly by researchers (Buckle and Kelly 1986; Griffith et al. 1987), these experiments mark the first attempt undertaken to specifically gain an insight into the bearing dynamic stability under realistic loading conditions. The four bearings (with properties listed in Table 1) supported a total gravity load of $226.86 \mathrm{kN}$ and were bolted to the base frame above and load cells below. The load cells were used to measure the horizontal and the vertical loads acting on each individual bearing. These four bearings used for dynamic testing were not subjected to the aforementioned quasi-static stability tests due to potential damage to the elastomer, hence the properties of the bearings from both tests vary slightly.

In the dynamic tests, the ground motions listed in Table 2 were applied at increasing intensity. The most intense ground motion proved to be the $85 \%$ MCE Erzincan record with the bearings exhibiting highly nonlinear behavior and pronounced excursions beyond their stability limits. The results from this particular test prove useful for calibration of the nonlinear analytical model of the bearing.

\section{Analytical model of dynamic test setup}

The dynamic test setup is modeled in the two-dimensional plane as a base isolated mass using 3 DOFs i.e. horizontal $(u)$, vertical $(v)$ and rotational $(\varphi)$. A schematic of the test setup and the simplified model is shown in Figure 4(a) and (b) respectively. The equations of motion of the system are derived based on equilibrium of the system in each direction.

$$
m_{1} \ddot{u}_{1}+c_{1} \dot{u}_{1}+k_{1} u_{1}=-m_{1}\left(\ddot{u}_{g}+\ddot{u}\right)-m_{1} h\left(\ddot{\varphi}_{g}+\ddot{\varphi}\right)
$$




$$
m \ddot{v}+2 c_{f} \dot{v}+2 k_{f} v=-m(\ddot{v}+g)
$$

190

191

192

An additional degree of freedom is introduced in the above equations corresponding to the mass $m_{l}$, this is done so as to incorporate the inertial term associated with this mass due to rotation. Hence $m_{b}$ refers to mass of base frame, $m_{l}$ refers to the mass of the steel plates and concrete blocks above the frame and $m$ refers to the total mass of the system. The equations of motion in the $\varphi$ direction are formulated about the centre of gravity of the base frame, $h$ hence refers to the distance between the centre of gravities of the base frame and mass $m_{l}$ located above it. $b$ refers to a half width between the bearings (for the setup used by Sanchez et al. (2012) the value is $1219.2 \mathrm{~mm}$ ). Since the total mass of the system is divided into two parts $m_{l}$ and $m_{b}$ only for convenience the stiffness associated with $m_{l}$ i.e. $k_{l}$ is assigned a very high value. In the above set of equations $I$ refers to the moment of inertia of base mat, $k_{f}$ and $c_{f}$ are the vertical stiffness and damping respectively of the bearings, $\ddot{u}_{g}$ and $\ddot{\varphi}_{g}$ are the acceleration input to the system measured using accelerometers placed on top of the shake table. The above systems of equations are solved using the unconditionally stable Newmark-Beta method.

The term $\tilde{k}_{h}$ refers to the combined horizontal stiffness of all four bearings of the system. It is a highly nonlinear term updated at each time step based on the magnitude of axial load acting on each bearing and the horizontal displacement. For calculation of the viscous damping coefficient $c_{b}$ of the bearings an average damping ratio of $3.3 \%$ is used, this value is chosen based on the effective damping ratios, $\beta_{\text {eff, }}$ of all the four bearings listed in Table 3. The specific focus of the study is to evaluate the ability of the nonlinear analytical model proposed above to model the observed experimental dynamic behavior. Since high nonlinear bearing behavior is anticipated, including considerable loss of stiffness beyond the stability limit, a corrective pseudo-force methodology developed by Nagarajaiah et al. (1991) is employed in the solution algorithm. The nonlinear forces corresponding to the bearings are represented separately as 
211 pseudoforces and at each time step an iterative corrective pseudoforces methodology is employed until

212 equilibrium is achieved and tolerance criteria are met.

213 Figure 5 shows a plan view of the test setup with identification label for each bearing. The input 214 excitation is applied in the east-west direction. An insight into the horizontal force-displacement, $F-u$, 215 behavior of the bearings is not possible without resolving the coupled horizontal-vertical behavior of the 216 bearings. Since the focus of this paper is only to gain an insight into the horizontal behavior, the vertical 217 dynamics are accounted for by using experimentally recorded values of vertical load at each of the 218 bearings. The coupled horizontal-vertical behavior of the bearings needs to be addressed, and is the 219 subject of further research. In this study, the nonlinear horizontal stiffness of each bearing is calculated at 220 every time step based on the experimental value of vertical load, $P$, recorded at that instant and the 221 horizontal displacement, $u$, calculated from the solution algorithm. Hence, when equations (6-9) are 222 solved, at each time step the vertical reaction at each bearing is updated based on experimentally recorded 223 values. The total horizontal force imparted by the four bearings is labeled $f_{b}$, the contributions from 224 bearings on the left side of the test setup (\#1 and \#3) are labeled $f_{b, l}$ and on the right side of the test setup $225\left(\# 2\right.$ and \#4) are labeled $f_{b, r}$.

\section{INPUT PARAMETERS}

228 The shear stiffness of the bearings at zero shear strain, $K_{s o}$, is obtained directly from experimental results 229 by differentiating the horizontal force, $F$, with respect to horizontal displacement, $u$, as shown below. In 230 order to obtain accurate values of $K_{s o}$ the horizontal force - displacement, $F-u$, curve obtained using 231 Method 2 for an axial load, $P$, of zero $\mathrm{kN}$ is chosen.

$$
K_{s o}=K_{h[\exp ]}(0, u)=\left.\frac{d F}{d u}\right|_{P=0}
$$



ground motion is shown in Figure 6. The difference in the stiffness of all four bearings is evident from this figure. The value of $K_{s o}$ for all the four bearings is estimated based on the horizontal force 236 displacement, $F-u$, curve for low values of $u$. From Table 3, the mechanical properties of the four 237 bearings used in the dynamic vary slightly, with, additional variation in stiffness due to the uneven 238 distribution of axial loads on the four bearings.

239 In an earlier study by Nagarajaiah and Ferrell (1999), the variation of shear modulus, $G$, was taken into 240 account using the dimensionless constant $C_{s}=0.325$. It is possible to estimate the value of $C_{s}$ with greater 241 accuracy due to the detailed experimental results available. The stiffness of the bearing can be determined 242 by differentiating $F$ (from Method 2 data for a constant value of $P$ ) with respect to $u$. At very small values 243 of $u$, the rotation of the analytical model is negligible. The main factor that governs the behavior of $K_{h}$ is 244 shear stiffness $K_{s}$, which in turn is dependent on the value of $C_{s}$. Figure 7 shows the accuracy of $C_{s}$ in 245 estimating the normalized stiffness curves compared to experimental values evaluated using horizontal 246 force - displacement, $F-u$, curves obtained from quasi-static test Method 2 for $P=0 \mathrm{kN}$. From Table 3

247 it can be seen that the value of $G_{\text {eff }}$ of bearing 15180 is high compared to all the other bearings (15196 248 and bearings $\# 1-4$ ) hence the value of $C_{s}=0.2821$ (determined from bearing 15196, Figure 7) is used 249 for the analytical model.

250 All the other input parameters of the nonlinear analytical model are calculated according to the following 251 relations (Buckle and Kelly 1986; Koh and Kelly 1986; Nagarajaiah and Ferrell 1999). The effective 252 flexural rigidity is calculated based on the approximation

$$
(E I)_{e f f}=E_{r} I \frac{l}{l_{r}}
$$

254 Where, $E_{r}$ is estimated as

$$
E_{r}=E_{o}\left(1+(2 / 3) S^{2}\right)
$$


256 The elastic modulus of rubber, $E_{o}=4 G_{o}, I$ is the moment of inertia of the bearing about the axis of 257 bending, and $S$ is the shape factor defined as

$$
S=\frac{\left(D_{o}-D_{i}\right)}{4 \times t_{r}}
$$

259 In the equation above, $D_{o}$ and $D_{i}$ are the outer diameter of the bearing and the diameter of the mandrel

260 hole and $t_{r}$ is the thickness of each rubber layer of the bearing.

$261 K_{\theta o}$ is estimated as follows

$$
K_{\theta o}=\frac{\pi^{2}(E I)_{e f f}}{l}=\frac{\pi^{2} E_{r} I}{l_{r}}
$$

The dimensionless constant, $C_{\theta}$, is estimated based on the physically motivated formula dependent on the rubber layer thickness of the bearings (Nagarajaiah and Ferrell 1999).

$$
C_{\theta}=\alpha C_{\theta}^{\prime}=l_{r}\left(\frac{t_{u}}{D_{o}}-\frac{t_{r}}{D_{o}}\right)
$$

267 where, $t_{u}$ refers to rubber layer of unit thickness, $l_{r}$ is the total thickness of the rubber and $\alpha$ is a dimensionless constant with a value of $l_{r}$.

\section{Adequacy of the Nagarajaiah and Ferrell (1999) model for dynamic loads}

The emphasis of an earlier study (Nagarajaiah and Ferrell 1999) was to develop an analytical model that

271 is able to capture the post-critical behavior of elastomeric bearings observed experimentally. In light of

272 the experimental results provided by Sanchez et al.(Sanchez et al. 2012), the ability of the Nagarajaiah

273 and Ferrell (1999) model to predict the response of the bearings is evaluated. Figure 8 shows the

274 simulated response of the bearings for $85 \%$ MCE Erzincan ground motion. It is evident that the analytical

275 model is not predicting the stability limit and stiffness degradation beyond this point accurately. The

276 stiffness of the bearings beyond the stability limit is greater than that predicted by the analytical model 
277 indicating that the bearings have additional reserve capacity to recover from instability. Nagarajaiah and

278 Ferrell (1999) model was based on quasi-static tests of bearings carried out under controlled loading

279 conditions, the results of the experiments presented here were carried out under dynamic conditions

280 where the bearings are free to move without being influenced by any predetermined loading condition.

\section{Proposed analytical model}

282 At large values of $u$, the governing factor for $K_{h}$ is the variation of $K_{\theta}$, this observation is in agreement with recent findings by Han et al. (2013). The current formulation where $K_{\theta}$ is defined as a linear function of $s / l_{r}$ is clearly not sufficient. Thus, the formulation is modified by incorporating higher order terms of $s / l_{r}$ and redefining $K_{\theta}$ as follows

$$
K_{\theta}=K_{\theta o}\left(1-C_{\theta}\left(\frac{s}{l_{r}}\right)-C_{\theta 1}\left(\frac{s}{l_{r}}\right)^{2}-C_{\theta 2}\left(\frac{s}{l_{r}}\right)^{3}\right)
$$

287

where $C_{\theta 1}$ and $C_{\theta 2}$ are dimensionless parameters. These parameters are estimated based on the response of the bearings to $85 \%$ MCE Erzincan ground motion. A three dimensional plot of stiffness of the bearings as a function of axial load, $P$, and horizontal displacement of the bearing, $u$, is shown in Figure 9.

Since all the four bearings differ in their properties, the input parameters corresponding to each of the bearing models are also varied accordingly. The input parameters of the new model proposed in this study are estimated based on the response of the bearings to Erzincan ground motion. The accuracy of the model is then verified using ground motion not considered for estimation of input parameters; namely Kobe and Newhall ground motion. For initial estimates of $K_{s o}$, data from $20 \%$ MCE Erzincan ground motion is used with the analytical model proposed by Nagarajaiah and Ferrell(1999). For estimating the dimensionless parameters $C_{\theta 1}$ and $C_{\theta 2}, 85 \%$ MCE Erzincan ground motion is considered along with the new analytical model proposed in this study. As described and demonstrated earlier in Figure 8 , the stiffness of the bearings beyond the stability limit predicted by Nagarajaiah and Ferrell (1999) model 
degrades too rapidly. A more gradual descent in stiffness is desired in order to better capture the response of the bearings both at and beyond the stability limit. The input parameters for all four bearings estimated based on Erzincan ground motion are listed in Table 4. In Figure 10 a comparison is made between the $K_{\theta}$ obtained from Nagarajaiah and Ferrell (1999) model and the new analytical model proposed above for a constant value of axial load, $P$, acting on the bearing.

Figure 11 shows the force - displacement, $F-u$, response of the bearings subjected to $20 \%$ MCE level of the Erzincan ground motion and Figure 12 shows the time history response of the forces experienced by the bearings and also the base displacement response predicted by the analytical model. Clearly the initial stiffness of the bearings is estimated well. Figure 13 and Figure 14 show the response of the bearings to $85 \%$ MCE level of ground motion. Simulation results are able to clearly capture the nonlinear reduction in stiffness associated with each bearing at and beyond the stability limits. In Figure 13, the drop in stiffness at the instant of instability is captured well; however, the loop width of the simulated response differs from that of experimental response indicating greater energy dissipation in the experiment.

During dynamic testing, Masroor et al. (2012) observed gradual and minimal change in the properties of the bearings as ground motion intensities are increased. The largest change was recorded occurred after the $85 \%$ MCE Erzincan input motion, indicating approximately 10\% drop in shear modulus and $13 \%$ increase in damping ratio of the bearings. These changes occurred because of damage to the bearings after reaching large strains beyond the instability limit in first cycle of the Erzincan ground motion. In this study those changes have not been deliberately incorporated, hence the stiffness of bearings differ slightly from observed experimental results (\#1 and \#4 bearings in Figure 13). This is the reason for the discrepancy in shear response prediction of bearings \#1 and \#4 observed from the time histories presented in Figure 14. In spite of these discrepancies, the base displacement is captured well using the new analytical model shown in Figure 14, especially the peak values. Under service conditions, bearing properties vary with time with some of these changes difficult to monitor. It is hence important to evaluate if the analytical model developed in this study is capable of predicting the response despite these 
small changes in their properties. The ability of the analytical model in capturing the response of the bearings to varying intensity levels of Erzincan ground motion is shown in Figure 15. For brevity, only two bearings (\#1 and \#2) are presented, since the other bearings experienced similar displacements and axial load variations as the bearings on the same side along the testing direction. The accuracy of the analytical model is demonstrated for various MCE levels of Erzincan ground motion.

\section{VERIFICATION OF MODEL FOR OTHER GROUND MOTIONS}

For verification purposes experimental results for Kobe and Northridge, Newhall ground motions are used. The results of the analytical model are presented next.

\section{Kobe ground motion}

Simulated response of the bearings for Kobe ground motion of intensity 20\%, 40\%, 67\% and 100\% MCE level are shown in Figure 16 for bearings \#1 and \#2. It can be seen that the stiffness of the bearings has been well estimated at all intensities of ground motion. The peak values of shear forces in all four bearings and the peak base displacements are also captured well as shown as the intensity of the ground motion increases from $20 \%$ to $100 \%$ MCE level. The closeness of the fit between predicted and experimentally observed response is demonstrated.

\section{Newhall ground motion}

Simulated response of bearings for Newhall ground motion for $20 \%, 40 \%$ and $100 \%$ MCE levels are shown in Figure 17 (for bearings \#1 and \#2). The analytical model predicts the response well at all ground intensities. The reduction in stiffness observed in the bearings for 100\% MCE level of Newhall ground motion is more pronounced when compared to its response for $100 \%$ Kobe ground motion and the analytical model is able to capture it well.

For comparison purposes, the response of the bearings for 100\% MCE level Kobe and Newhall ground motions are simulated using the Nagarajaiah and Ferrell (1999) model and presented for bearings \#1 and 
\#2 in Figure 18. It is evident that the earlier model proposed by Nagarajaiah and Ferrell (1999) is unable to model the response accurately once the bearing reaches the stability limits. In Figure 19 the quasi-static stability curves are plotted along with the dynamic response of the bearings to $85 \%$ MCE Erzincan ground motions. Force - displacement, $F-u$, curves obtained from bearing 15196 using quasi-static test Method 2 for axial loads 44.48 and $88.96 \mathrm{kN}$ are presented in Figure 19 and compared to the dynamic response of bearing (bearing \#1 for $85 \%$ MCE Erzincan) with axial load variation that lies within this range. It is clear from the plot that the available quasi-static test data only provide information up to the stability limit.

\section{DiscuSSION AND CONCLUSION}

357 From the experimental results presented, it is clear that the stiffness degradation of the elastomeric 358 bearings beyond the stability limit is not predicted accurately by earlier models (Nagarajaiah and Ferrell 1999). The analytical model proposed in this study clearly captures the observed behavior of the bearings

360 at lower intensities with very high accuracy. At higher intensities (85\% MCE Erzincan) where the 361 behavior of the bearing becomes highly nonlinear in nature, despite the difference in properties of the four 362 bearings the analytical model predicts with reasonable accuracy the critical load and captures the response 363 for the entire extent of loss of stability of the bearings. The unique experimental results available 364 combined with the current analytical model provide a detailed insight into the nonlinear behavior of the 365 bearings. When the response of the bearings to the most intense ground motions is considered, it becomes apparent that the bearings exhibit significant capacity to sustain loads far beyond the static stability limit.

367 Another important conclusion from this study is that in order to accurately capture the behavior of the 368 bearings beyond the stability limit, analytical model parameters derived from quasi-static tests are 369 insufficient. The dimensionless parameters $C_{\theta 1}$ and $C_{\theta 2}$ are crucial for predicting the response of the 370 bearings observed beyond the stability limit and their values cannot be determined based on quasi-static 371 tests alone. Though extensive experimental findings are presented in this and earlier studies by co-authors 372 of this study, results from bearings of different geometry need to be evaluated using the current model 
before any general conclusions regarding the input parameters can be reached. In summary, the analytical model presented in this study gives valuable insight into the nonlinear behavior of bearings and represents the first attempt to model the nonlinear dynamic response for the entire displacement range including the region beyond the stability limit.

\section{ACKNOWLEDGEMENTS}

Authors VVSM and SN gratefully acknowledge funding support provided by grant NSF-CMMI-NEESR-0830391 for this project. AM and GM were supported by NSF-NEESR-CMMI-1113275.

\section{REFERENCES}

Bažant, Z. P. (2003). Shear Buckling of Sandwich, Fiber Composite and Lattice Columns, Bearings, and Helical Springs: Paradox Resolved. Journal of Applied Mechanics, 70 (1), 75-83.

Bažant, Z. P., \& Cedolin, L. (1991). Stability of Structures. New York: Oxford University Press.

Buckle, I., \& Kelly, J. M. (1986). Properties of slender elastomeric isolation bearings during shake table studies of a large-scale model bridge deck. Joint Sealing and bearing systems for concrete structures , 247-269.

Buckle, I., Nagarajaiah, S., \& Ferrell, K. (2002). Stability of Elastomeric Isolation Bearings: Experimental Study. J. Struct. Engrg. , 128 (1), 3-11.

Cardone, D., \& Perrone, G. (2012). Critical load of slender elastomeric seismic isolators: An experimental perspective. Engineering Structures , 198-204.

Derham, C. J., \& Thomas, A. G. (1981). In Control of seismic response of piping system and other structures. (pp. 21-36).

Gent, A. N. (1964). Elastic stability of rubber compression springs. J. Mech. Engrg. Sci. , 6 (4), 318-326.

Griffith, M. C., Kelly, J. M., \& Aiken, I. D. (1987). A displacement control and uplift restraint device for base isolated devices. UCB/EERC-87/3, University of California, Berkeley, Berkeley, CA.

Han, X., Kelleher, C. A., Warn, G. P., \& Wagener, T. (2013). Identification of the controlling mechanism for predicting critical loads in elastomeric bearings. J. Struct. Engrg. , DOI: 10.1061/(ASCE)ST.1943-541X.0000811.

Haringx, J. A. (1948). On highly compressible helical springs and rubber rods and their application for vibration-free mountings. I. Phillips Res. Rep. , 3, 401-449.

Haringx, J. A. (1949). On highly compressible helical springs and rubber rods and their application for vibration-free mountings. II. Phillips Res. Rep., , 4, 49-80.

Haringx, J. A. (1949). On highly compressible helical springs and rubber rods and their application for vibration-free mountings. III. Phillips Res. Rep. , 4, 206-220.

lizuka, M. (2000). A macroscopic model for predicting large-deformation behaviors of laminated rubber bearings. Engineering Structures, 323-334.

Kikuchi, M., Nakamura, T., \& Aiken, I. D. (2010). Three-dimensional analysis for square seismic isolation bearings under large shear deformations and high axial loads. Earthquake Engng Struct. Dyn. , 39, 1513-1531. 
Koh, C. G., \& Kelly, J. M. (1988). A simple mechanical model for elastomeric bearings used in base isolation. International Journal of Mechanical Sciences, 30 (12), 933-943.

Koh, C. G., \& Kelly, J. M. (1986). Effects of axial load on elastomeric bearings. University of California, Berkeley. Berkeley, CA: Earthquake Engineering Research Center.

Koh, C. G., \& Kelly, J. M. (1989). Viscoelastic Stability Model for Elastomeric Isolation Bearings. J. Struct. Engrg. , 115 (2), 285-302.

Masroor, A., Sanchez, J., Mosqueda, G., \& Ryan, K. L. (2012). Dynamic Stability of Elastomeric Bearings at Large Displacement. Proceedings of 15th World Conference on Earthquake Engineering. Lisbon, Portugal.

Nagarajaiah, S., \& Ferrell, K. (1999). Stability of Elastomeric Seismic Isolation Bearings. J. Struct. Engrg. , 125 (9), 946-954.

Nagarajaiah, S., Reinhorn, A. M., \& Constantinou, M. C. (1991). Nonlinear dynamic analysis of 3-d-baseisolated structures. J. Struct. Engrg. , 117 (7), 2035-2054.

Sanchez, J. (2010). Stability of elastomeric bearings for seismic applications. MS Thesis, Department of Civil, Structural and Environmental Engineering, State University of New York, Buffalo.

Sanchez, J., Masroor, A., Mosqueda, G., \& Ryan, K. L. (2012). Static and Dynamic Stability of Elastomeric Bearings for Seismic Protection of Structures. J. Struct. Engrg. , DOI: 10.1061/(ASCE)ST.1943541X.0000660.

Warn, G. P., \& Weisman, J. (2011). Parametric finite element investigation of the critical load capacity of elastomeric strip bearings. Engineering Structures , 33 (12), 3509-3515.

Weisman, J., \& Warn, G. P. (2012). Stability of Elastomeric and Lead-Rubber Seismic Isolation Bearings. J. Struct. Engrg. , 138 (2), 215-223. 


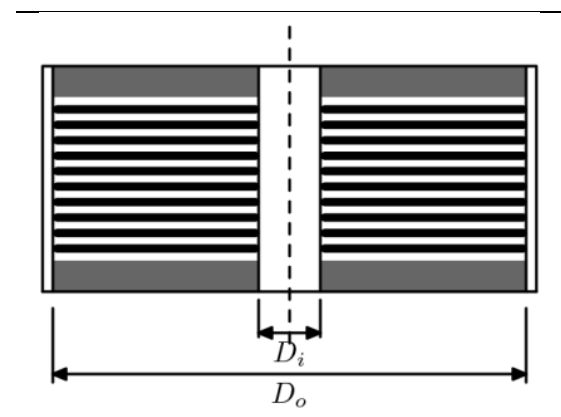

\begin{tabular}{lr} 
Shape Factor, $S$ & 10.64 \\
\hline Height $(\mathrm{mm})$ & 163.07 \\
\hline Outside diameter, $D_{o}(\mathrm{~mm})$ & 165.1 \\
\hline Inside diameter, $D_{i}(\mathrm{~mm})$ & 29.97 \\
\hline Thickness of rubber, $t_{r}(\mathrm{~mm})$ & 3.175 \\
\hline Number of rubber layers, $n_{r}$ & 25
\end{tabular}

\begin{tabular}{lr}
\hline Area, $A_{b}=\pi / 4\left(D_{o}^{2}-D_{i}^{2}\right)\left(\mathrm{mm}^{2}\right)$ & 20702.9 \\
\hline$G_{\text {eff }}$ at $25 \%(\mathrm{MPa})$ & 0.60 \\
\hline$G_{\text {eff }}$ at $100 \%(\mathrm{MPa})$ & 0.46
\end{tabular}

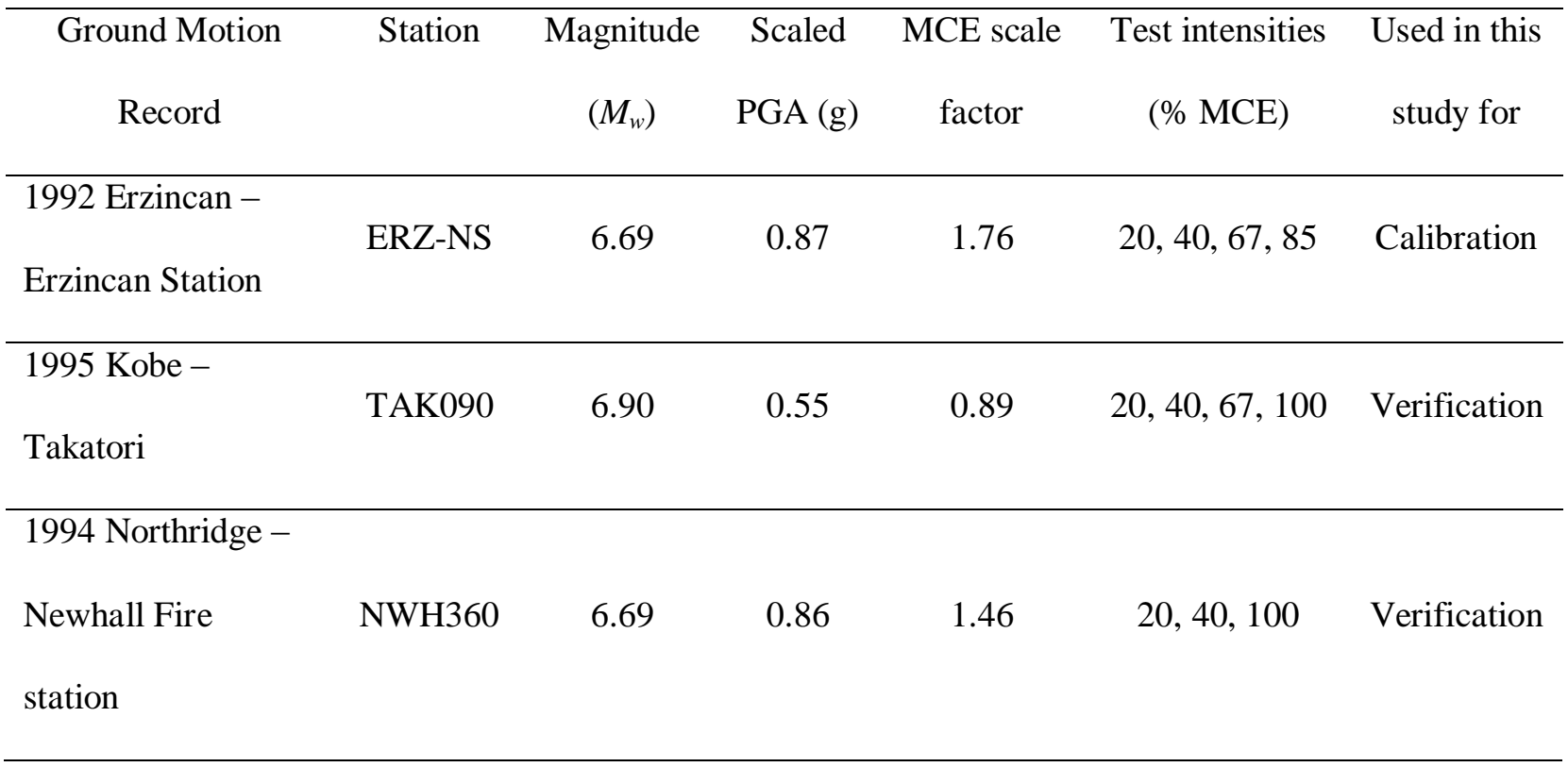

447 Table 3: Effective shear modulus, $G_{\text {eff }}$, and damping ratio, $\beta_{\text {eff }}$, at $100 \%$ shear strain for bearing used in experimental study. 


\begin{tabular}{cccccc}
\hline & & \multicolumn{2}{c}{$G_{\text {eff }}(\mathrm{MPa})$} & \multicolumn{2}{c}{$\beta_{\text {eff }}(\%)$} \\
\cline { 1 - 2 } \multicolumn{2}{c}{ Axial Load (kips) } & 10 & 14 & 10 & 14 \\
\cline { 1 - 2 } Test Type & Bearing ID & & & & \\
\cline { 1 - 2 } Quasi Static & 15196 & 0.531 & 0.476 & 3.2 & 3.8 \\
Quasi Static & 15180 & 0.579 & 0.524 & 2.8 & 3.1 \\
Dynamic & $\# 1-\mathrm{NW}$ & 0.524 & 0.386 & 3.2 & 3.4 \\
Dynamic & $\# 2-\mathrm{NE}$ & 0.545 & 0.414 & 3.1 & 3.3 \\
Dynamic & $\# 3-\mathrm{SW}$ & 0.524 & 0.407 & 3.2 & 3.4 \\
Dynamic & $\# 4-\mathrm{SE}$ & 0.531 & 0.407 & 3.1 & 3.2 \\
\hline
\end{tabular}

449

450

Table 4: Parameters for the four bearings in the dynamic tests for the new analytical model

\begin{tabular}{cccc}
\hline & $C_{\theta 1}$ & $C_{\theta 2}$ & $K_{s o}$ \\
\cline { 1 - 2 } Original values & -0.0977 & 0.0136 & $(\mathrm{kN} / \mathrm{mm})$ \\
\cline { 1 - 2 } & \multicolumn{2}{l}{ Multiplication factors } & \\
\cline { 1 - 2 } 2 & 1.00 & 1.00 & 0.1869 \\
3 & 1.12 & 1.33 & 0.1682 \\
4 & 1.28 & 1.22 & 0.1159 \\
& 0.88 & 1.11 & 0.2468 \\
\hline
\end{tabular}

451

452 


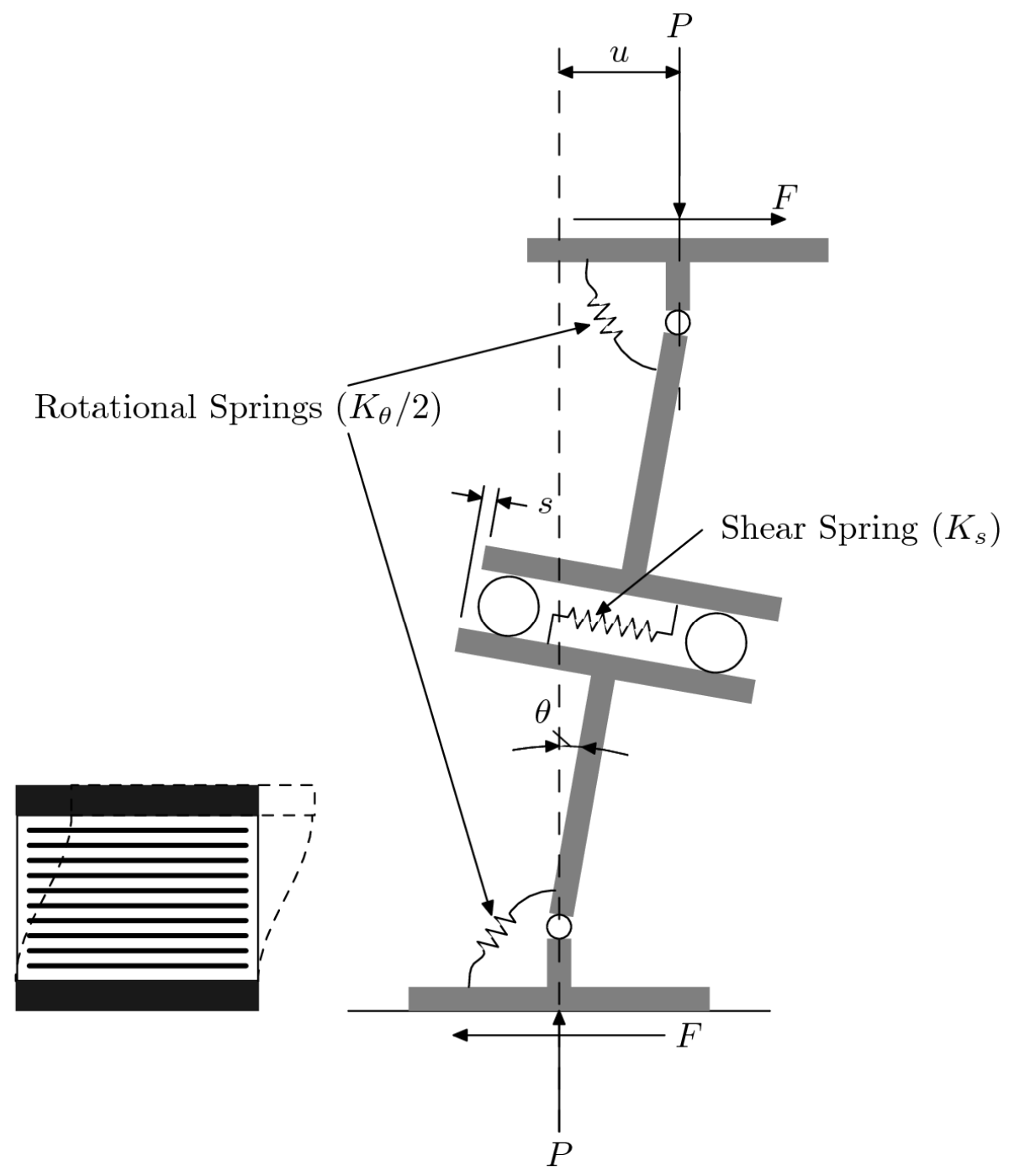

(a) Elastomeric bearing (b) Analytical model (not to scale) 
Click here to download Figure: figure 2.pdf

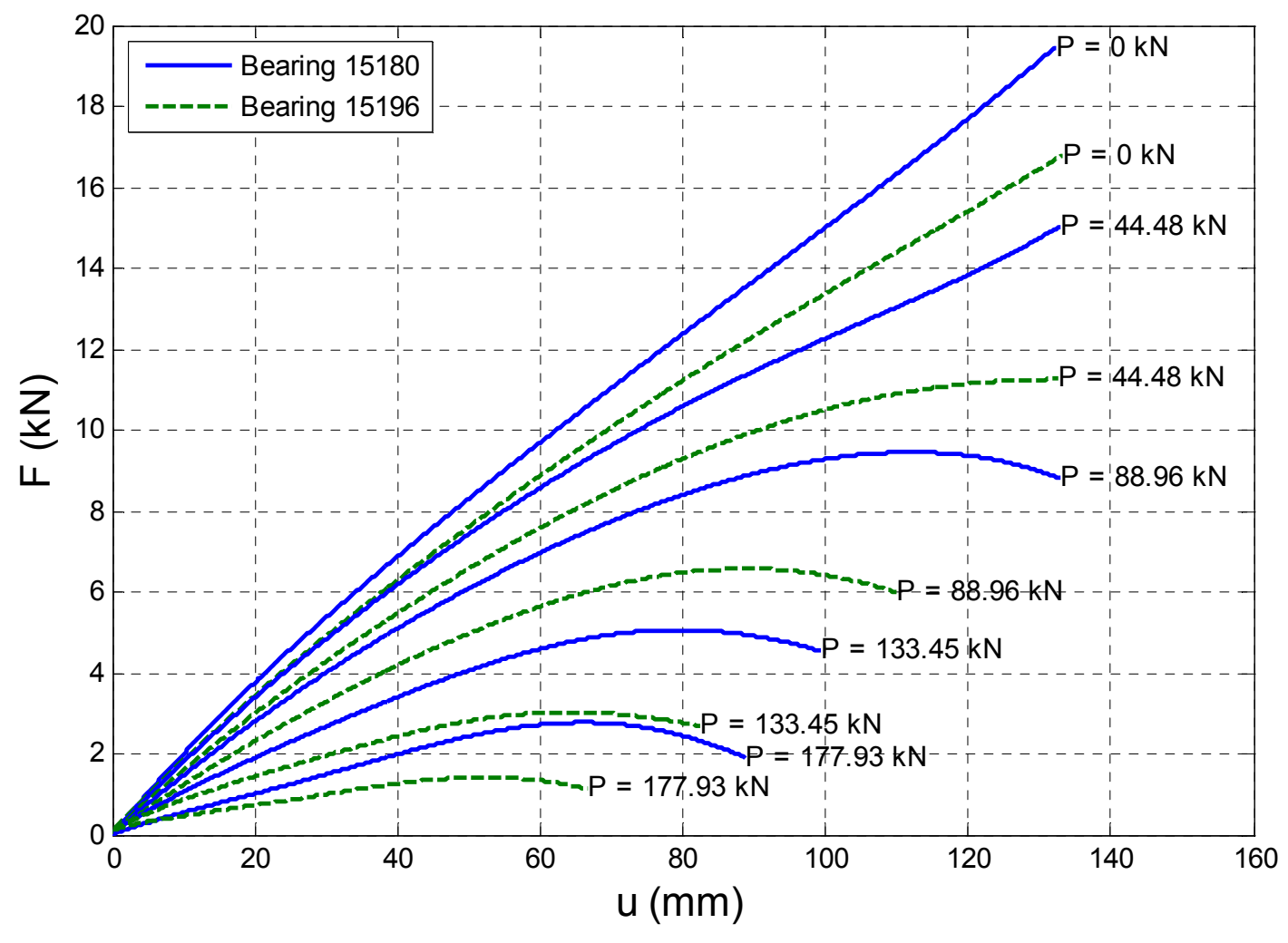




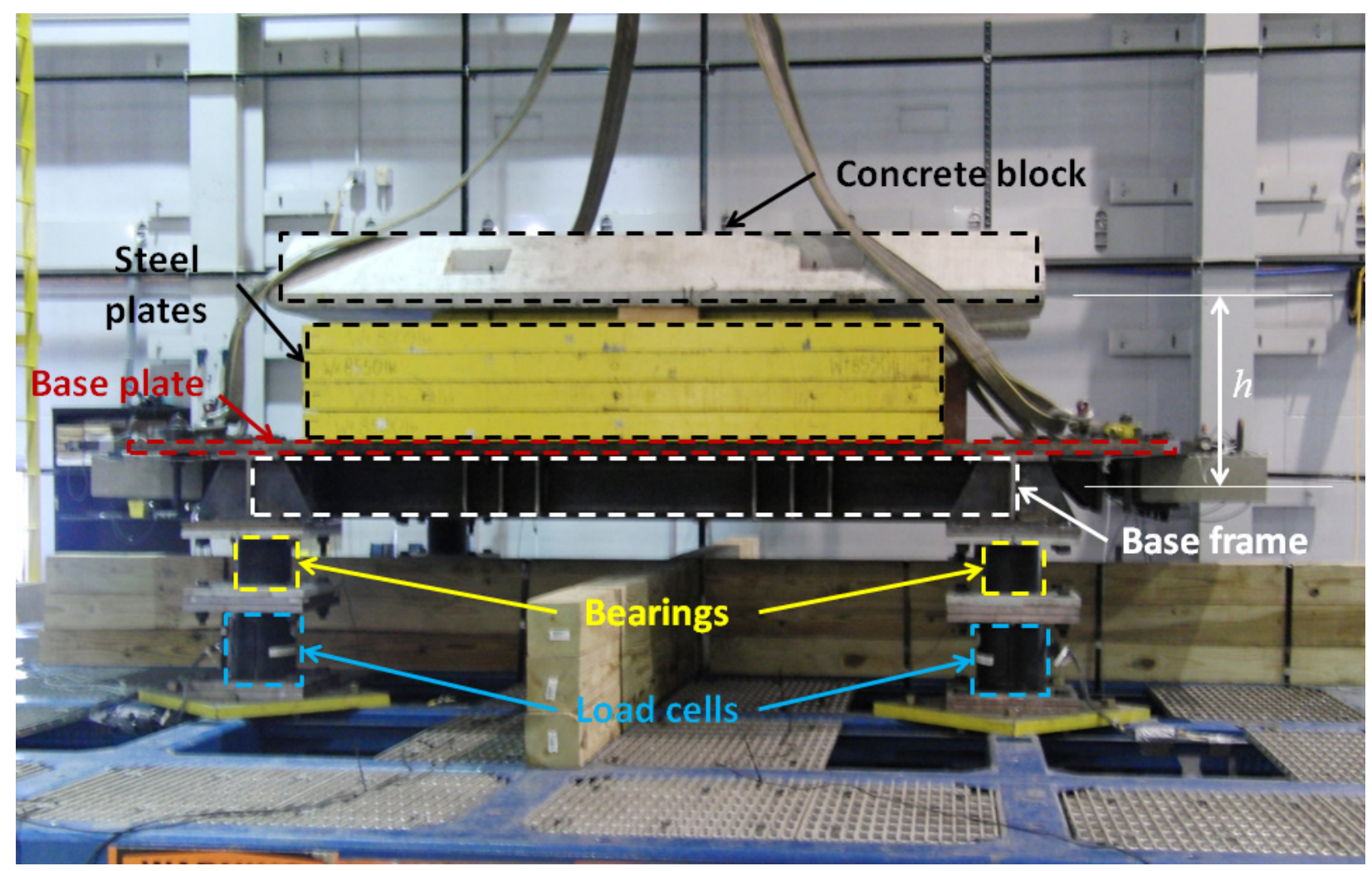




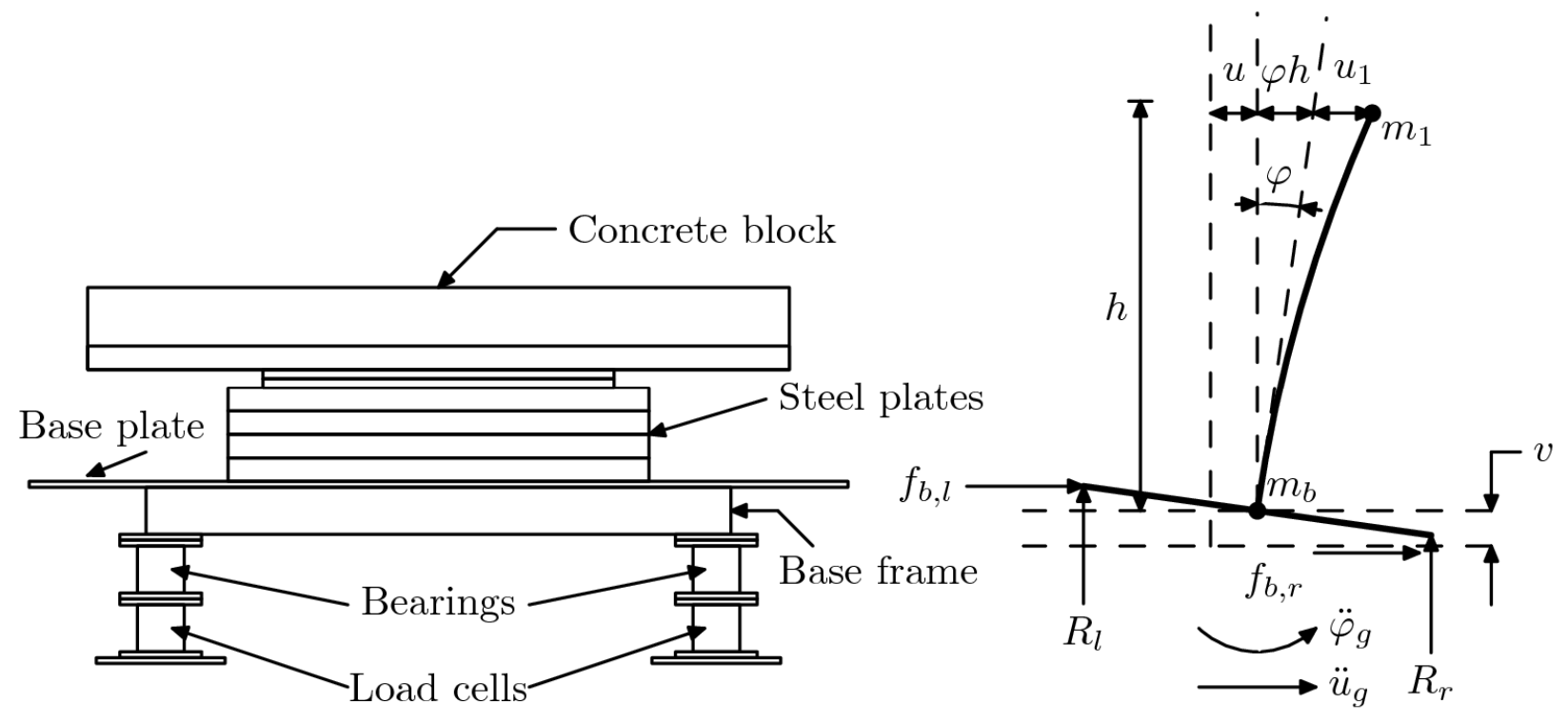

(a) Test setup

(b) Deformed model(not to scale) 
Figure
Click here to download Figure: figure 5.pdf

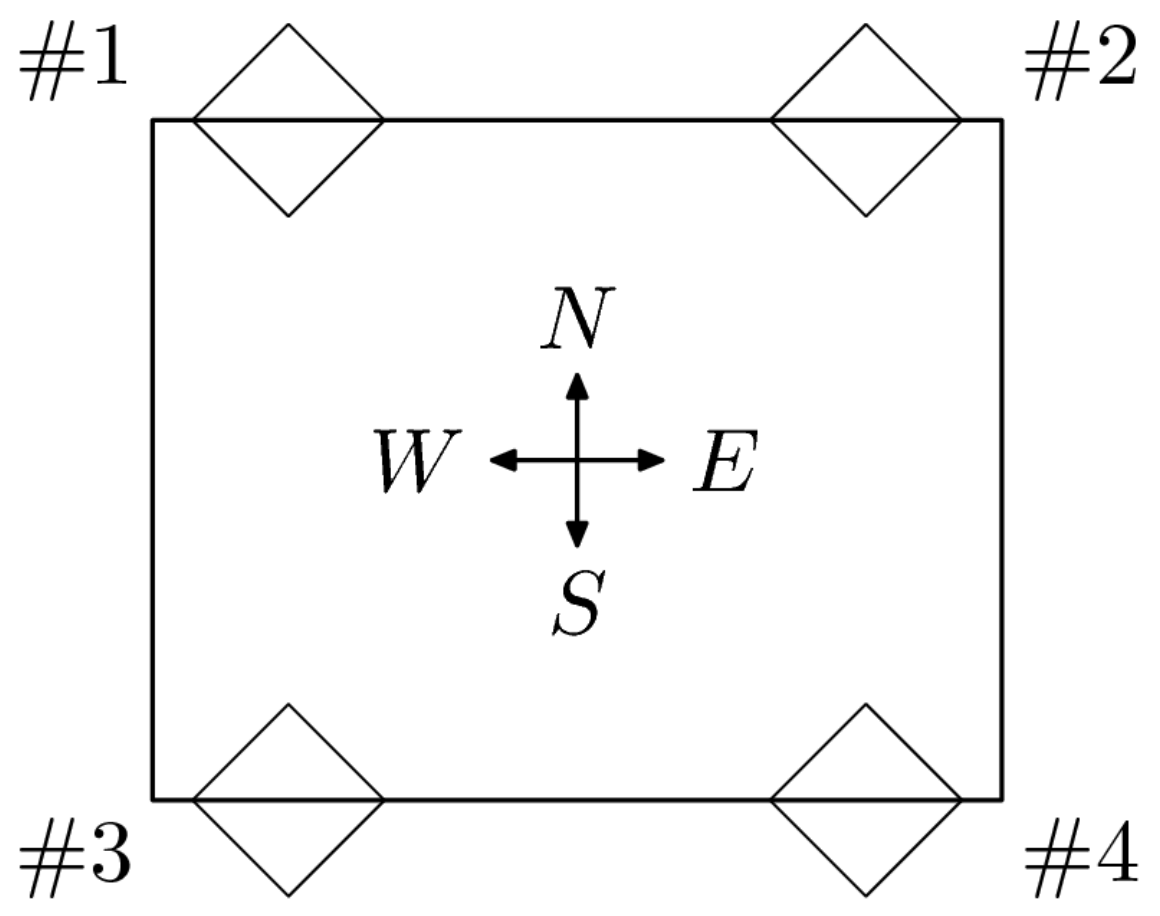



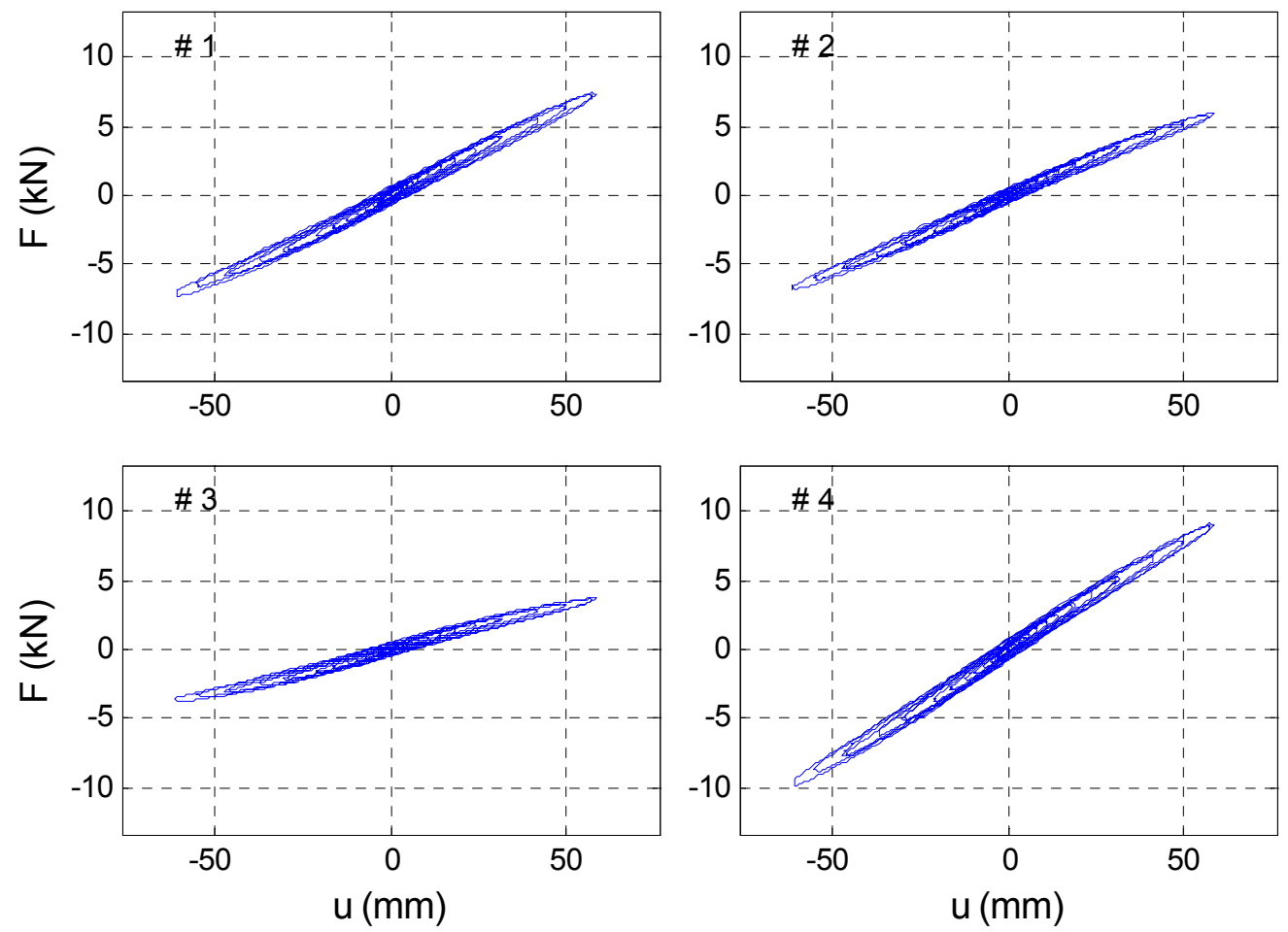

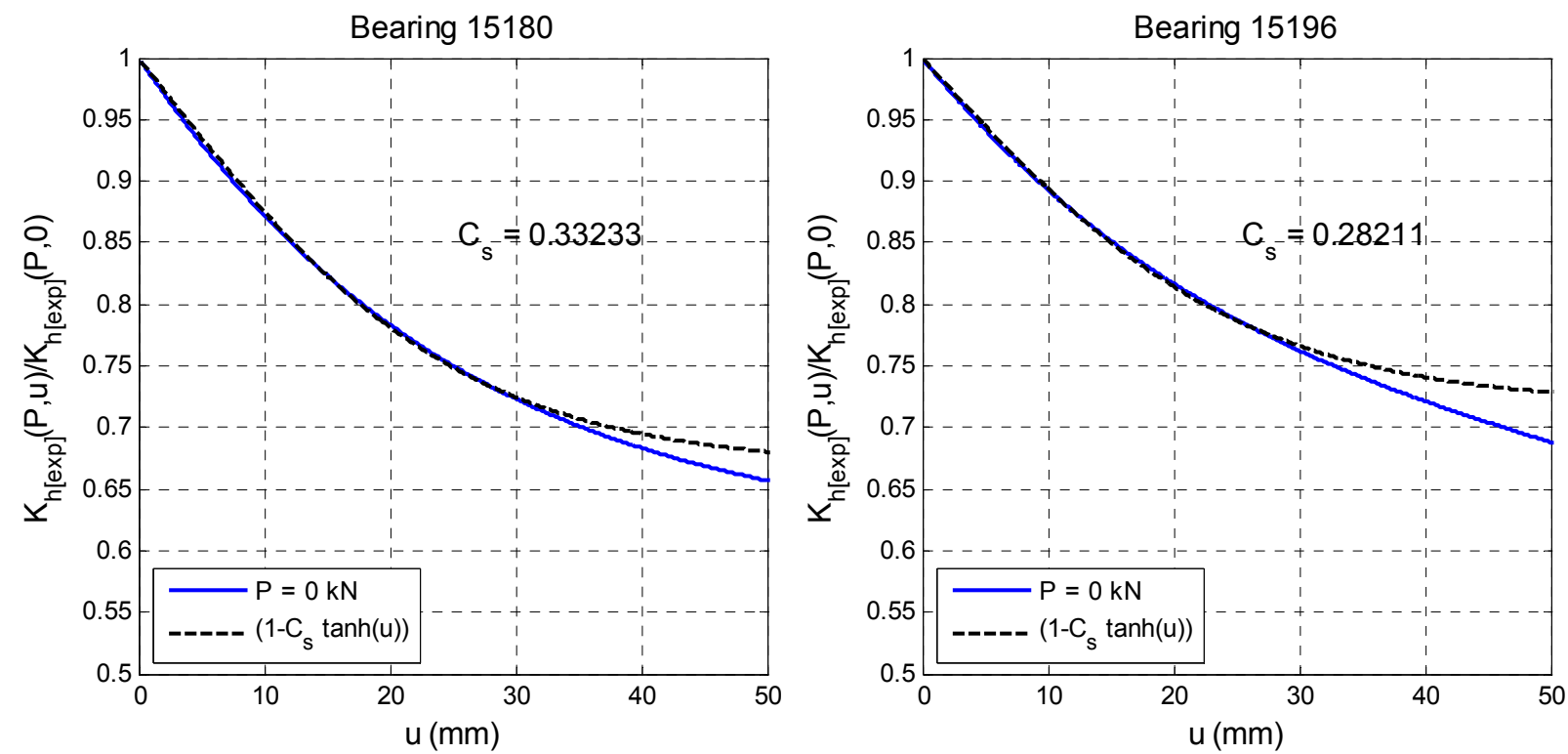




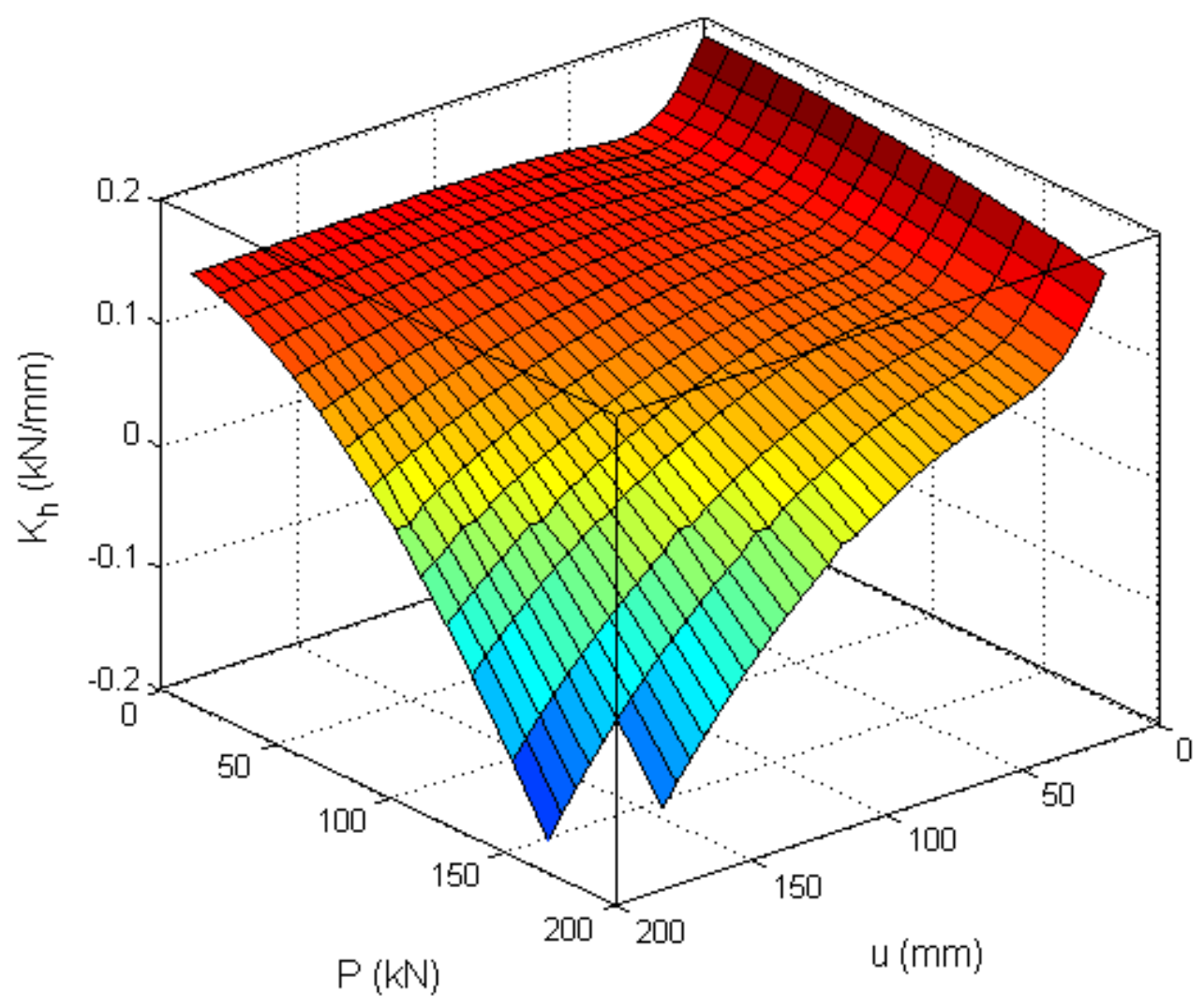




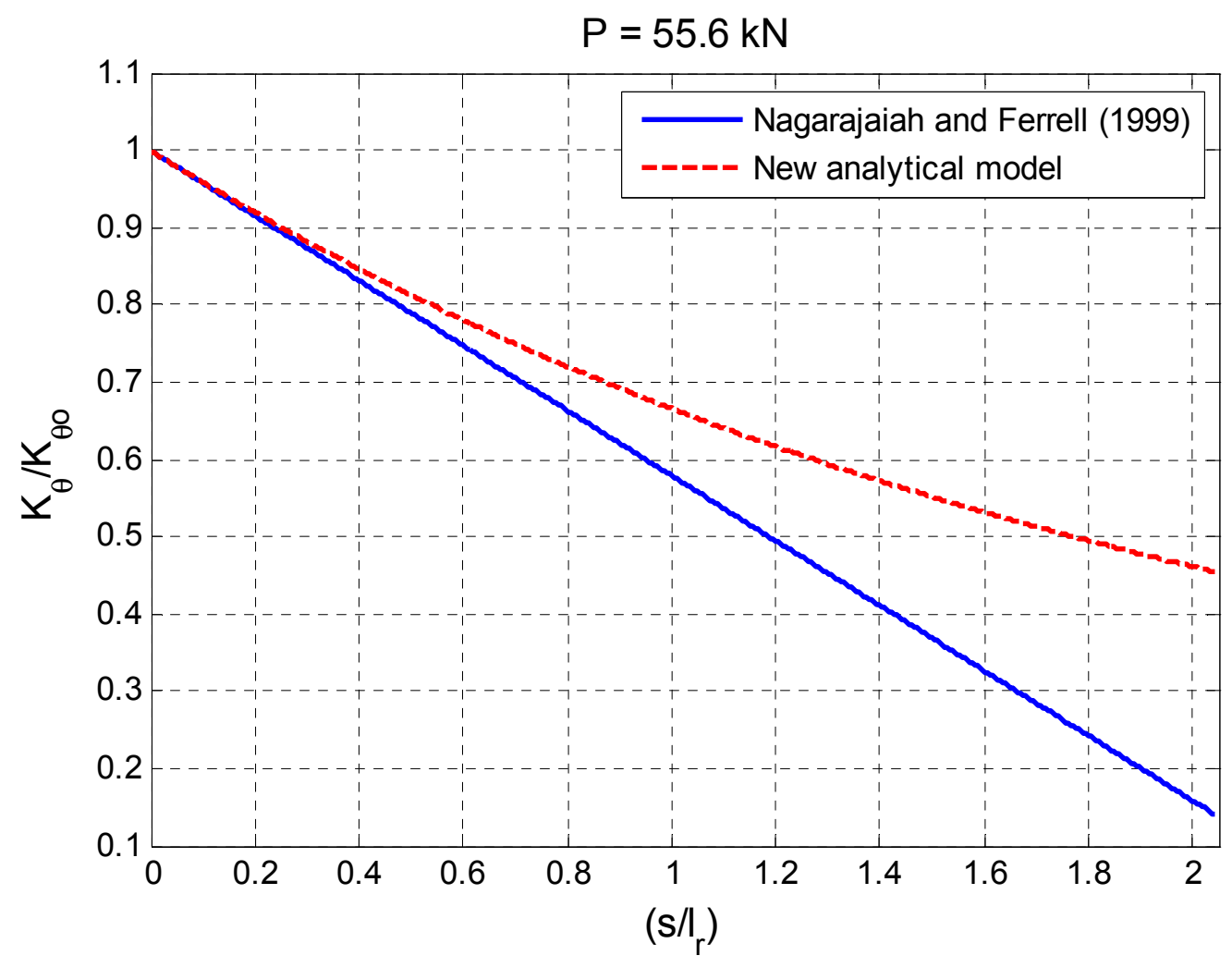



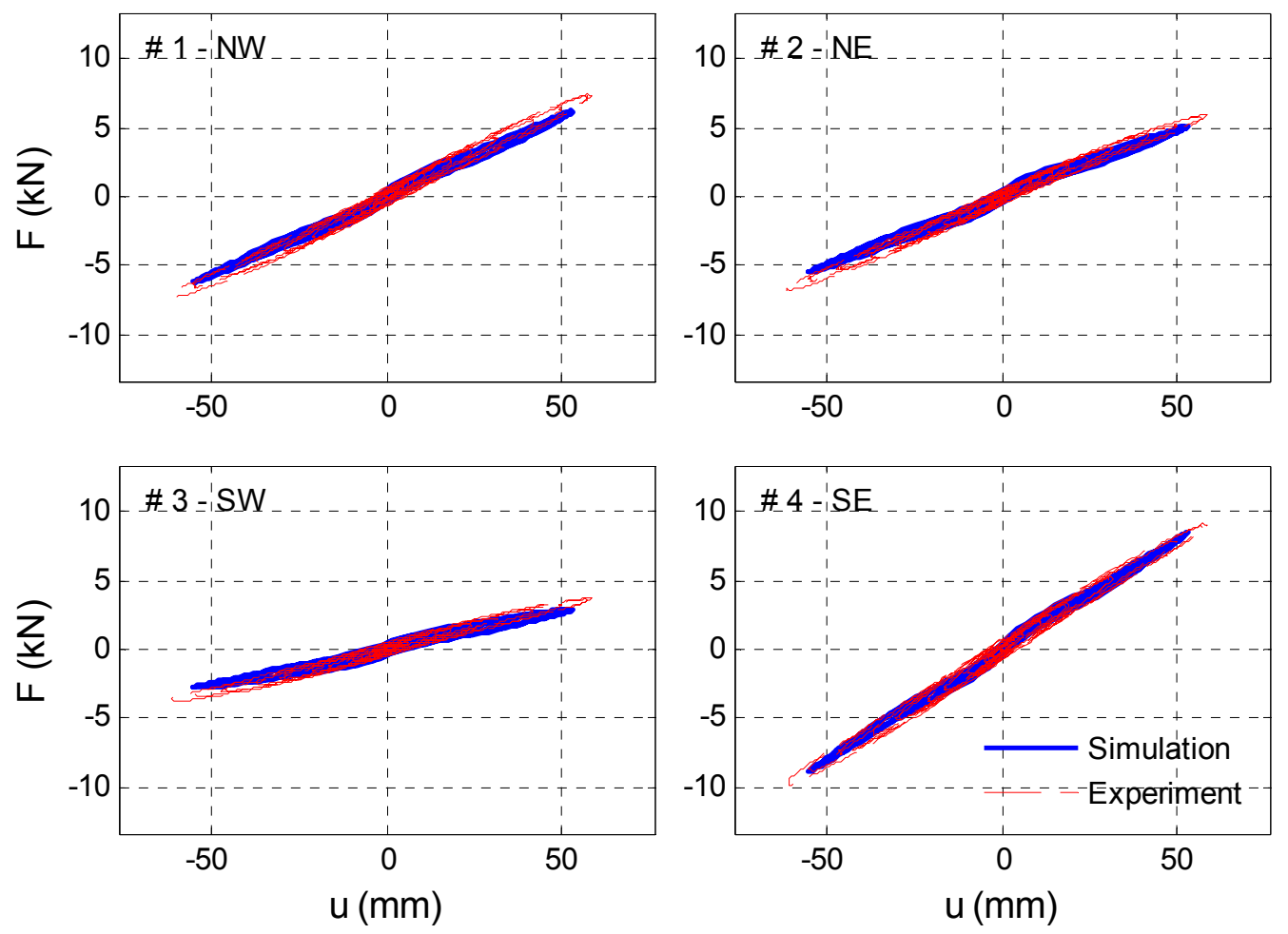

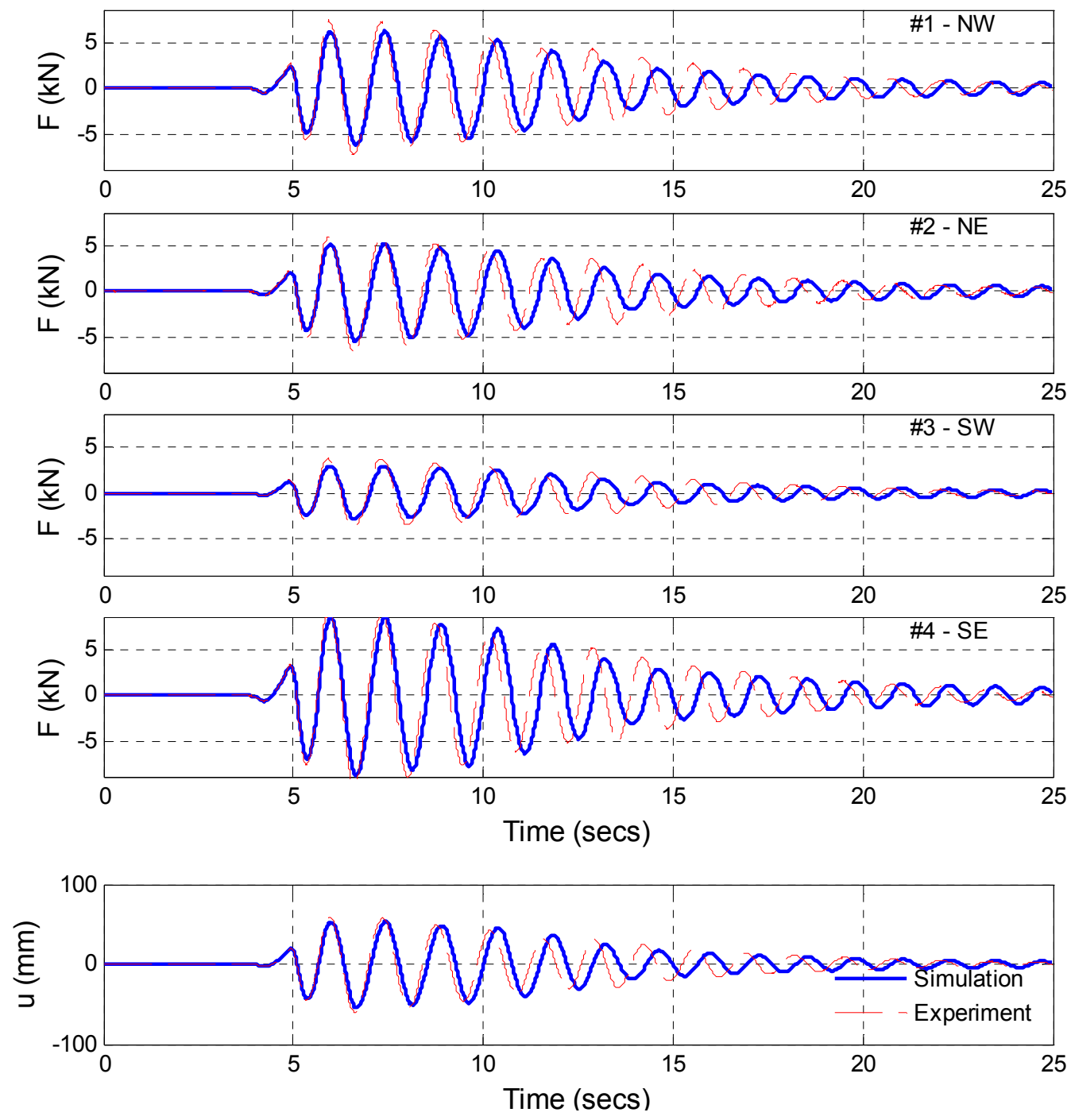

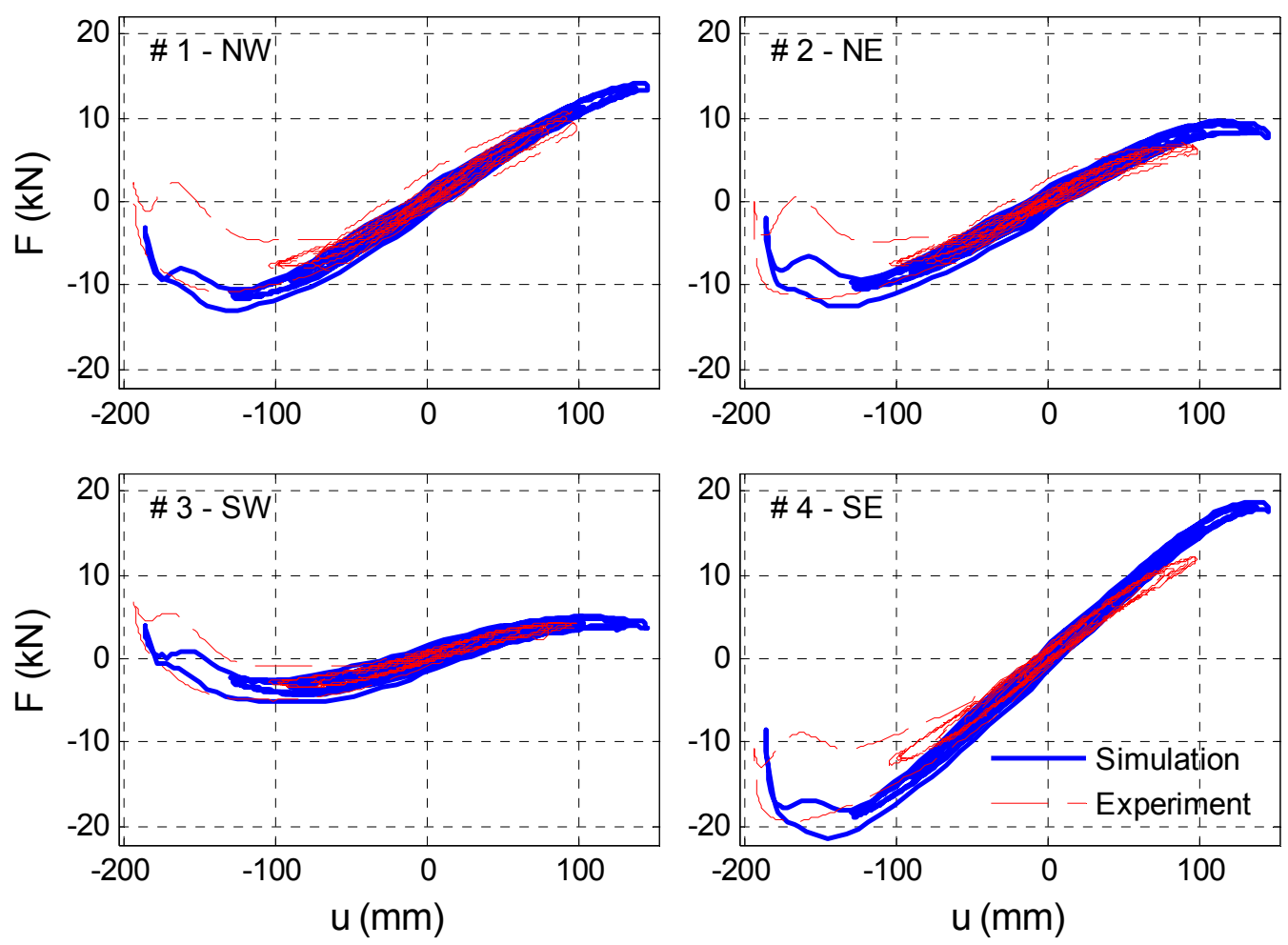

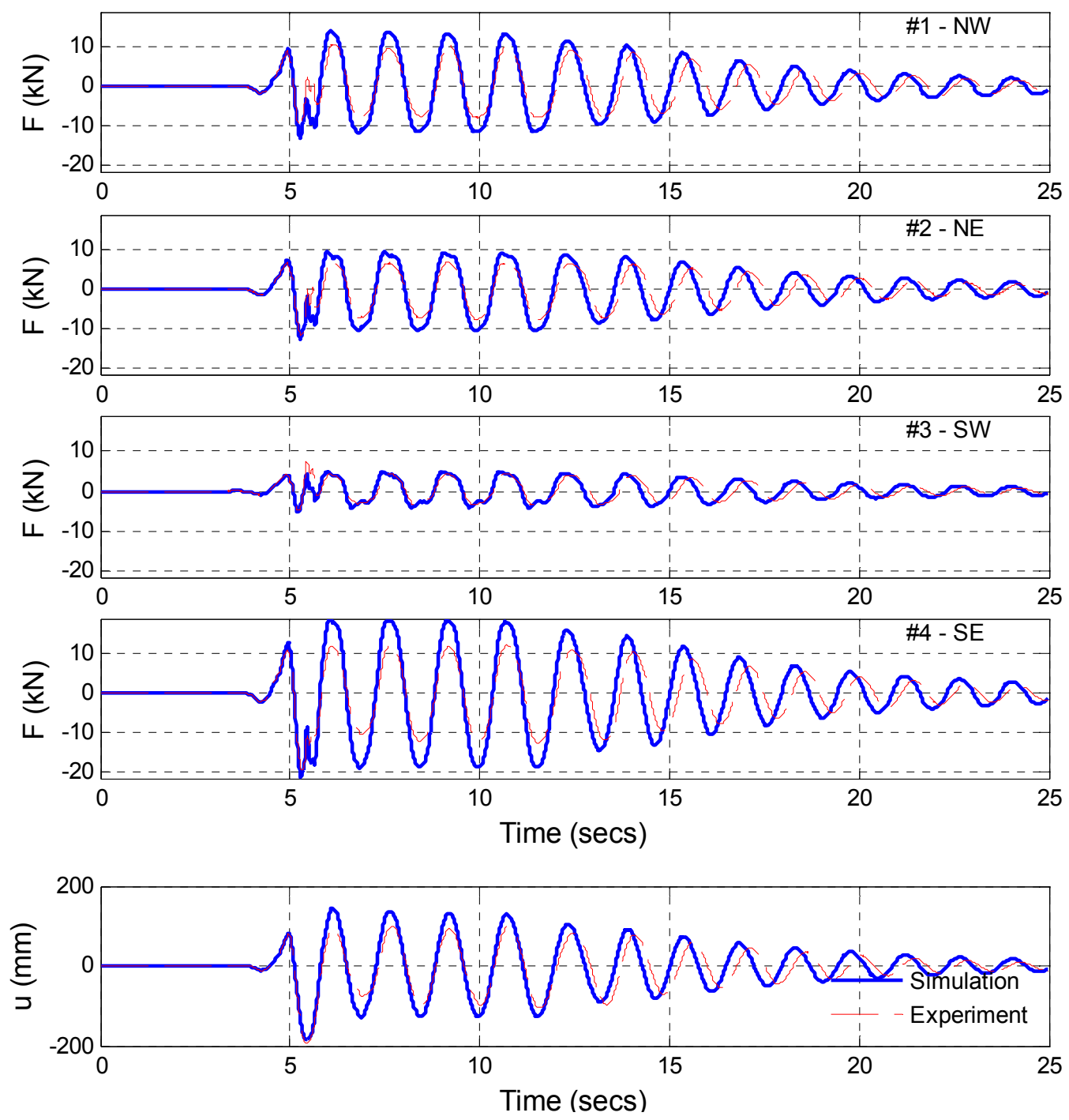

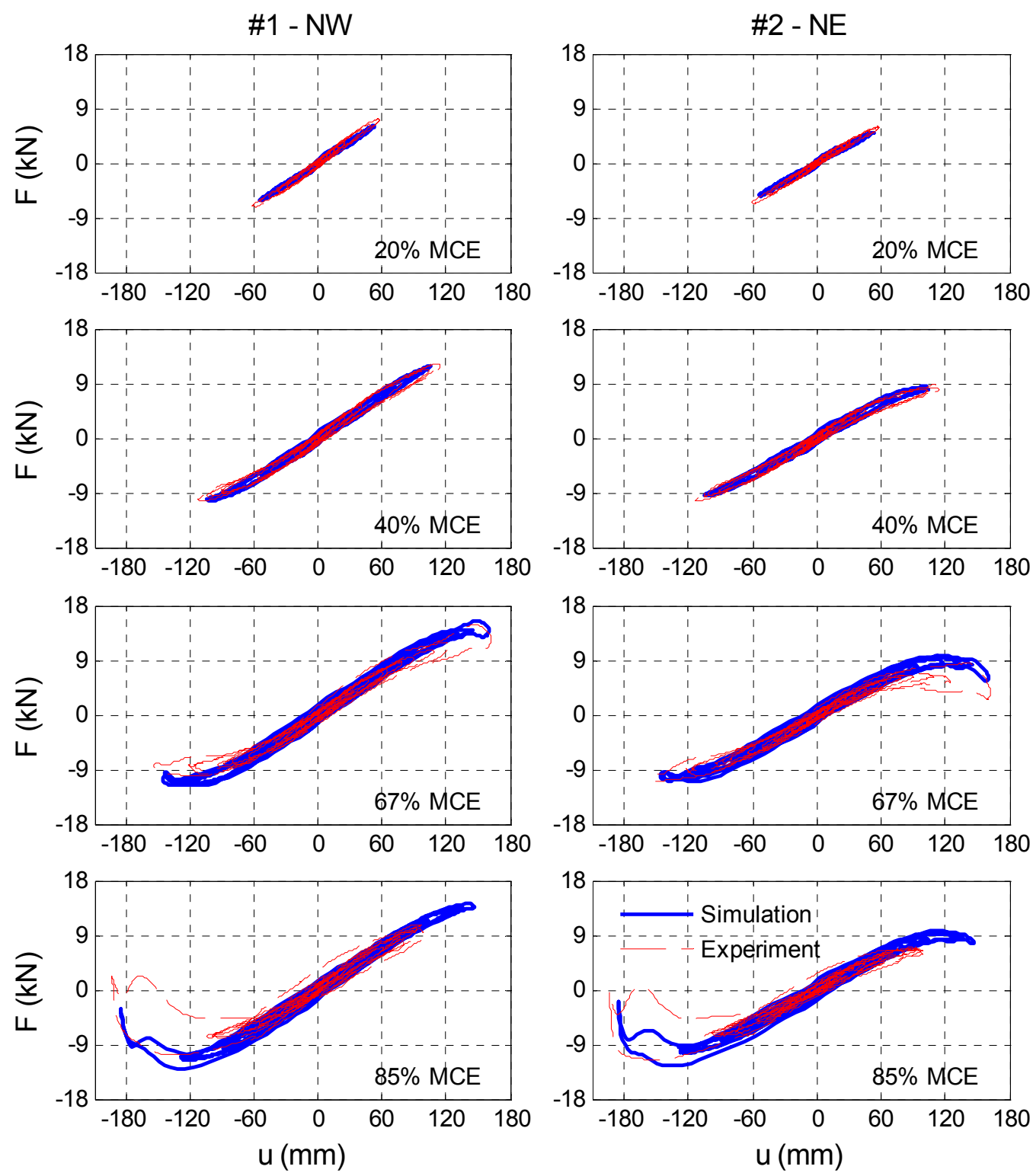

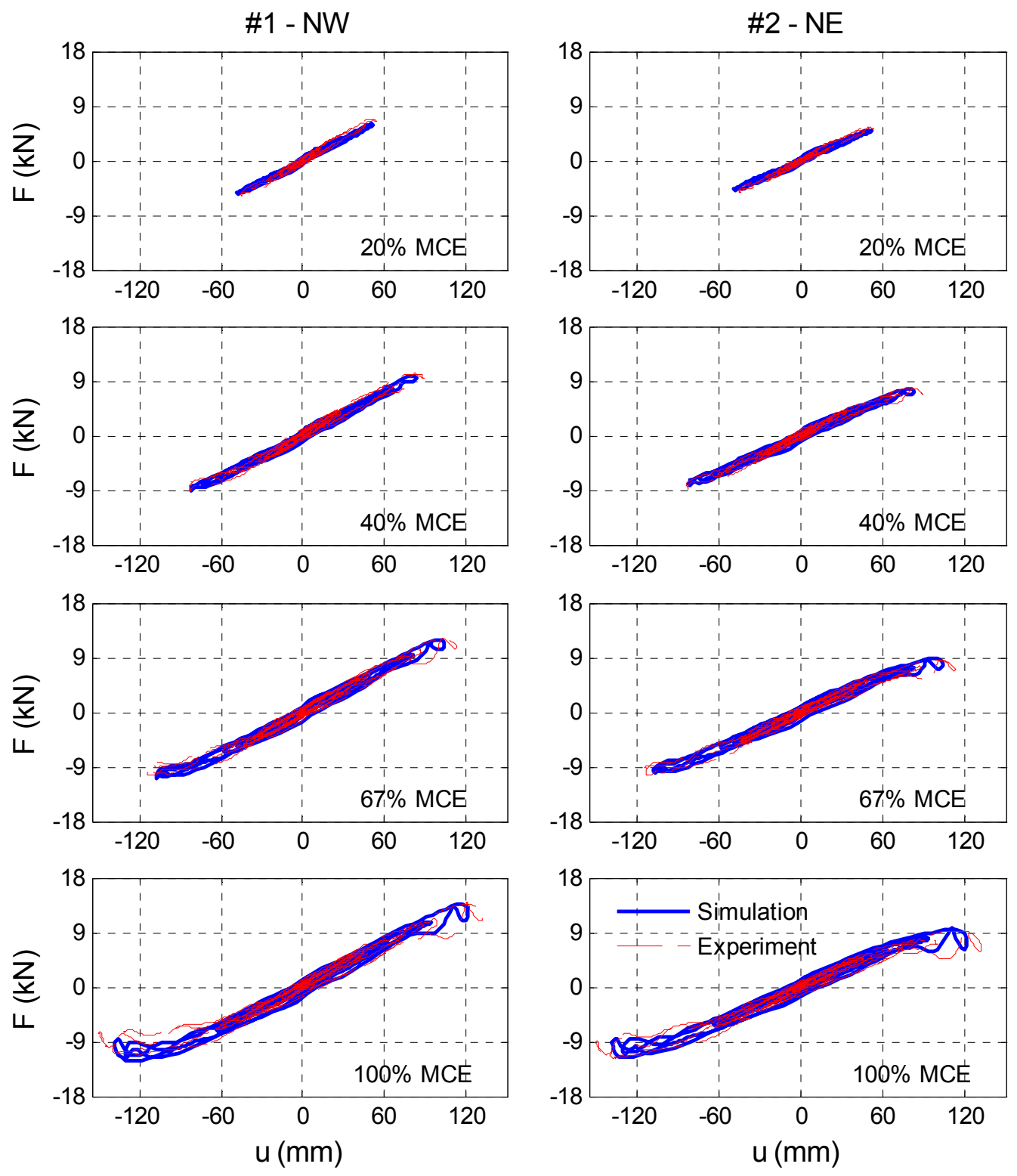

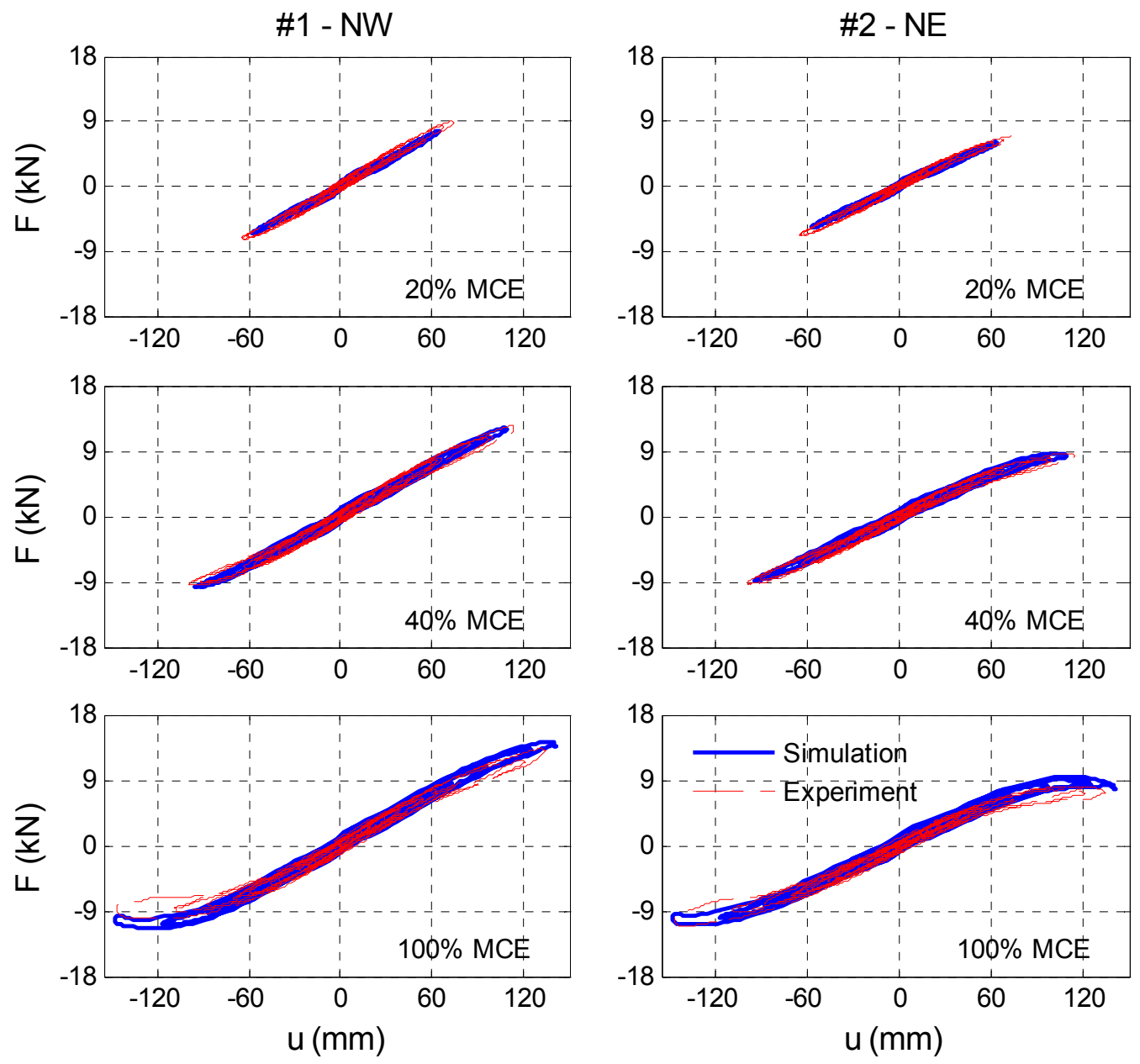

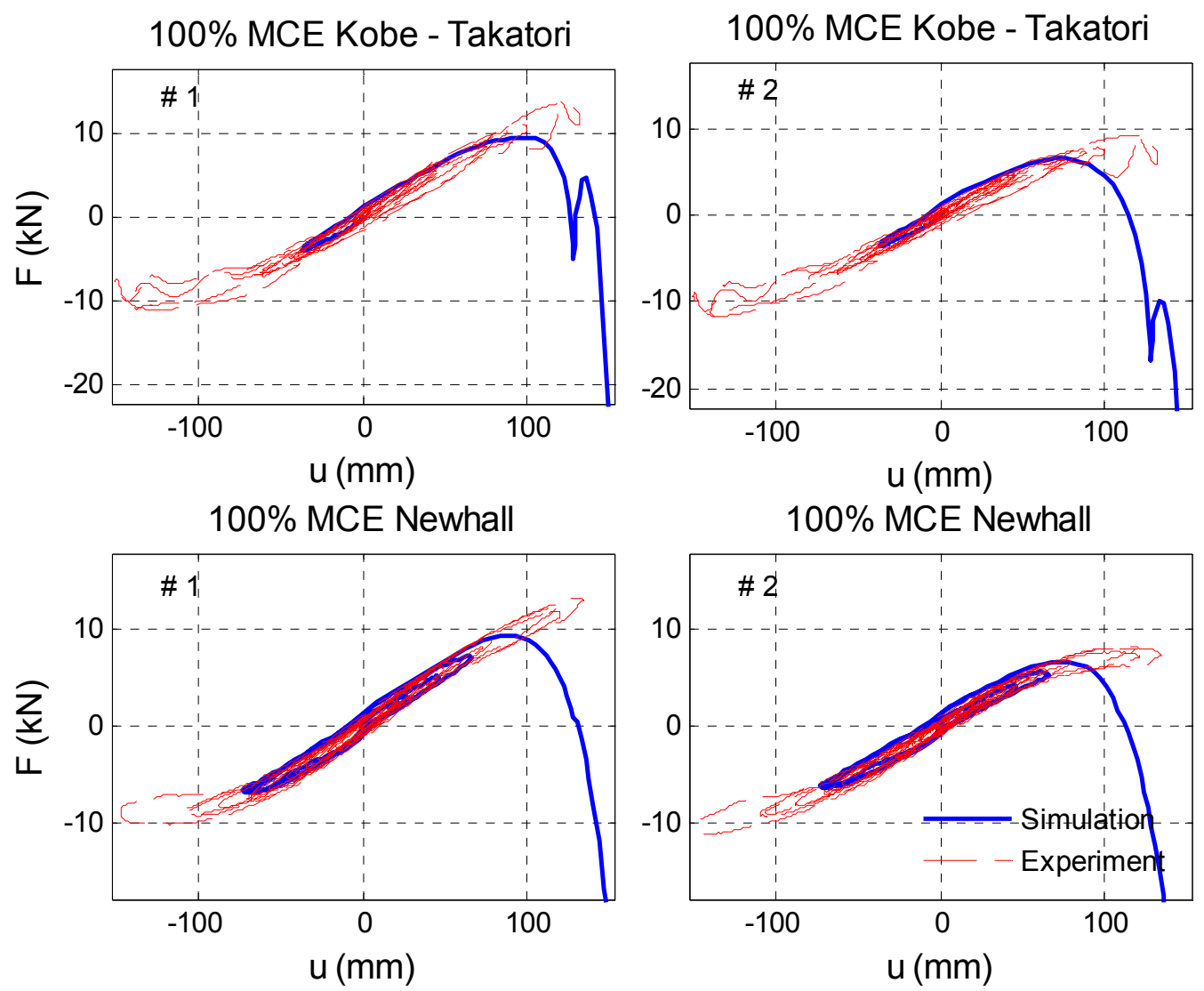


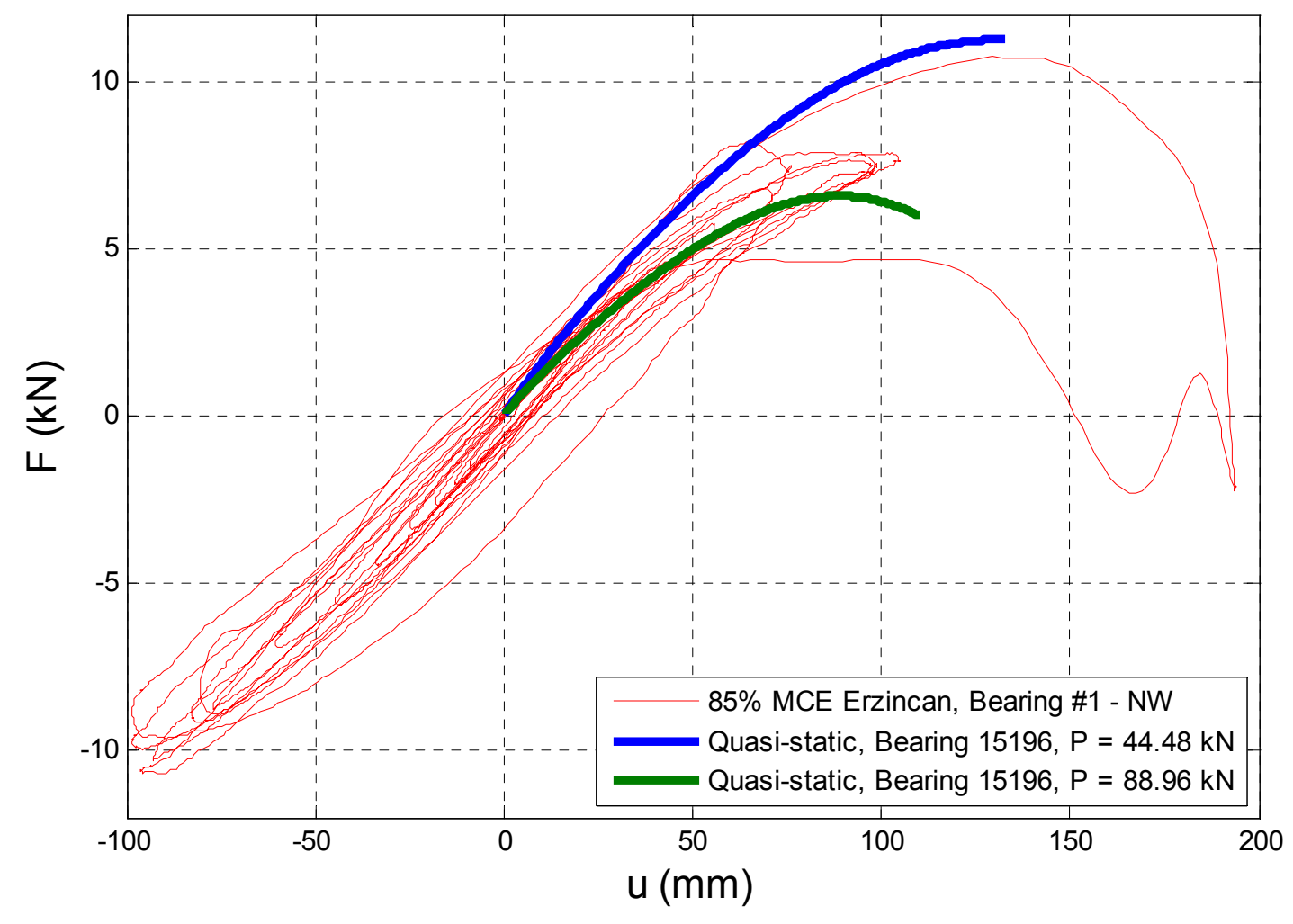


Figure 1: Nonlinear analytical model used in this study

Figure 2: Force - displacement behavior of two different bearings (15180 and 15196) obtained by quasistatic stability test Method 2.

Figure 3: Test setup used for dynamic loading of bearings

Figure 4: Schematic of the experimental setup and analytical model used to simulate its response Figure 5: Top view of the test setup

Figure 6: Experimental force - displacement, $F-u$, response of the four bearings subjected to $20 \%$ MCE Erzincan ground motion

Figure 7: Normalized horizontal stiffness as a function of horizontal displacement obtained experimentally from horizontal force - displacement, $F-u$, curves for $P=0 \mathrm{kN}$ and analytically based on estimated value of $C_{s}$ for bearings 15180 and 15196.

Figure 8: Simulated horizontal force - displacement, $F-u$, response of the bearings using model by Nagarajaiah and Ferrell (1999) when subjected to 85\% MCE Erzincan ground motion

Figure 9: Three dimensional plot of stiffness of the bearing, $K_{h}$, as a function of axial load, $P$, and horizontal displacement, $u$, generated using the new analytical model.

Figure 10: Normalized plot of rotational stiffness, $K_{\theta}$, as a function of shear displacement, $s$, obtained from the model by Nagarajaiah and Ferrell (1999) and the new analytical model

Figure 11: Simulated horizontal force - displacement, $F-u$, response of the bearings using new analytical nonlinear model when subjected to $20 \%$ MCE Erzincan ground motion

Figure 12: Simulated time histories of horizontal force, $F$, in all the four bearings and base displacement, $u$, using new analytical nonlinear model when subjected to 20\% MCE Erzincan ground motion

Figure 13: Simulated horizontal force - displacement, $F-u$, response of the bearings using new analytical nonlinear model when subjected to $85 \%$ MCE Erzincan ground motion 
Figure 14: Simulated time histories of horizontal force, $F$, in all the four bearings and base displacement, $u$, using new analytical nonlinear model when subjected to $85 \%$ MCE Erzincan ground motion

Figure 15: Simulated and experimental horizontal force - displacement, $F-u$, loops of bearings \#1 and \#2 when subjected to $20 \%, 40 \%, 67 \%$ and $85 \%$ MCE level Erzincan ground motion.

Figure 16: Simulated and experimental horizontal force - displacement, $F-u$, loops of bearings \#1 and $\# 2$ when subjected to $20 \%, 40 \%, 67 \%$ and $100 \%$ MCE level Kobe - Takatori ground motion.

Figure 17: Simulated and experimental horizontal force - displacement, $F-u$, loops of bearings \#1 and \#2 when subjected to $20 \%, 40 \%$ and $100 \%$ MCE level Newhall ground motion.

Figure 18: Simulated and experimental horizontal force - displacement, F- u, loops of bearings \#1 and \#2 when subjected to $100 \%$ MCE level Kobe and Newhall ground motions using the Nagarajaiah and Ferrell (1999) model.

Figure 19: Response of Bearing \#1 to 87\% Erzincan ground motion and quasi-static force - displacement, $F-u$, curves from bearing 15196 for axial loads of 44.48 and $88.96 \mathrm{kN}$. 